\title{
Research on a New Process of Agile Turn with Engine Reignition Based on Optimal Control
}

\author{
Kang Niu (D, Jianqiao Yu, Xi Chen, Di Yang, and Ziyuan Li \\ Department of Astronautics, Beijing Institute of Technology, Beijing 100081, China \\ Correspondence should be addressed to Kang Niu; astro_nuaank@163.com
}

Received 21 April 2021; Revised 1 September 2021; Accepted 12 September 2021; Published 12 November 2021

Academic Editor: Thach Ngoc Dinh

Copyright (c) 2021 Kang Niu et al. This is an open access article distributed under the Creative Commons Attribution License, which permits unrestricted use, distribution, and reproduction in any medium, provided the original work is properly cited.

\begin{abstract}
A new process of agile turn with engine reignition is proposed in this paper. Compared with the traditional process, this process includes deceleration phase, larger angle manoeuvre phase, and engine reignition phase. Firstly, the paper describes the new process of agile turn. Then, several constraints in this process are described. Considering all these constraints and assumptions, a new dynamic model including two-stage engine and deceleration parachute is established. Then, the optimal control laws are designed and the timing point determination of the secondary engine ignition is discussed. By using Pontryagin principle, the optimal control laws for each actuator are derived. In terms of determining the optimal timing point of the secondary engine ignition, the paper gives the process of proof. Finally, several numerical simulations are given to demonstrate the effectiveness of the method proposed in this paper. According to all these numerical simulations, it is obvious that the new process of agile turn proposed in this paper is better than traditional process especially in having a smaller turning radius, a shorter turning time, and a high terminal velocity.
\end{abstract}

\section{Introduction}

With the instant development of world militarily, agile turn is widely used in tactical and strategic weapons, especially in air-air missile, ground-to-air missile, and others. In air combat, to seize the transient opportunity, it requires pilots to have the ability of random launch. At the same time, the missile should have the ability of agile turn. As the counteraction becoming more and more fierce, the manoeuvrability of the moving target becomes so stronger that the traditional process of agile turn is not adapted to the current air combat mode. Beyond that, to attack the concealed targets in urban or the reverse side of the mountain, the missile should not only have the stronger manoeuvrability comparing with the traditional process of agile turn but also complete the turning process in a shorter time. In addition, to ensure attacking the moving target, it is usually that the missile should have a high terminal velocity after great manoeuvring. All these problems challenge traditional agile turn process. So, how to realize supermanoeuvrable turning with having a high terminal velocity, a smaller turning radius, and decreasing the time in turning phase and others has become a research hotspot in the field of missile.

As is known to all, the angle between the orientation of velocity and LOS (Line of Sight) is very large when launching missile. With the effectiveness of initial guidance, the missile pointing can be soon adjusted to near LOS. This will be instrumental in terminal guidance. In terms of agile turn, there are too many literatures about it. In the early research, TVC (Thrust Vector Control) and RCS (Reaction jet Control System) had been widely used in USA [1]. These two kinds of methods are investigated to provide direct control force and moment for missile. Reference [2] focuses on how to control the orientation of velocity and a new method combining with RCS is proposed in this paper. At the same time, considering the property of RCS, a sliding mode control method is used for attitude control. In terms of using direct lateral force, considering multiple constraints for agile turn, a turning law employing the optimal axis control principle is designed in [3] and several numerical simulations are given to realize it. To improve the accuracy of a control system, a parameter selection method for lateral thrust and 
aerodynamic force is introduced in [4], but the influence of instruction form is not considered in this method. To avoid the limit cycles and blended control logic problem, a new control logic about RCS method is designed in [5]. Several simulations show the effectiveness of this method, but it just considers the problem of blended control logic. In [6], the agile turn problem was formulated as an optimal control problem, and the optimization goal is having a max terminal velocity during the whole phase. In [7], a novel extended modal series method for solving the infinite horizon optimal control problem of nonlinear interconnected large-scale dynamic systems is presented. In this method, the infinite horizon nonlinear large-scale two-point boundary value problem (TPBVP), derived from Pontryagin's maximum principle, is transformed into a sequence of linear timeinvariant TPBVPs. In [8], a new guidance law for initial turn manoeuvre is presented. In this paper, a backstepping controller is designed to follow the attack angle command generated from the guidance model. While this method divides this process into two phases of turning and acceleration, each phase is analysed separately. In [9], a new method for solving optimal guidance problems is proposed via the theory of connections. This paper applies this approach to solve the boundary value problems for differential equations to solve optimal space guidance problems. To solve the optimal control problem of linear time-varying systems with state time-delay, a recursive shooting method is presented in [10]. Firstly, the original time-delay optimal control problem is transformed into a sequence of linear two-point boundary value problems (TPBVPs) without delay and advance terms. Then, by using a shooting method for the solution of latter sequence in a recursive manner, an optimal control law is achieved which consists of feedback and forward terms. At last, several comparative results are included to illustrate the effectiveness of the proposed approach. Reference [11] uses the particle swarm optimizing method to design the control law for agile turn. Several numerical simulations had been done to verify the effectiveness, but this method is not better viewed from the turning radius. Reference [12] deals with a general form of fractional optimal control problems involving the fractional derivative with singular or nonsingular kernel. A new numerical method with Mittag-Leffler kernel is designed to solve these equations effectively. Above all these methods based on optimal control theory, there are many other approaches based on sliding control method. In [13], in terms of the canard control that may saturate at large angles, an integrated fuzzy sliding mode control method is developed for missile. This method using the double-sliding mode, zero miss-distance, and a variable related to autopilot parameter are selected as two sliding surfaces. Even though using this method can derive the appropriate control law, it is just suitable for snake maneuverer. Based on sliding mode control method and adaptive synchronization, a new adaptive sliding mode disturbance-observer in a class of fractional-order chaotic systems is designed in [14]. Using the Lyapunov stability theory, the designed controller fulfills that the states of the fractional-order master and slave chaotic systems are synchronized hastily. Several simulations demonstrate the efficiency of the offered scheme on the fractional-order Dadras-Momeni chaotic system in the existence of external disturbances. Reference [15] uses the computational fluid dynamics (CFD) method to analyze the influence of interaction flow field with multilateral jets, when attacking the target at large angles. The paper reveals the mechanism of the jet interaction effect on the aerodynamic characteristics of the missile at different attack angles, and several simulations verify the effectiveness of this method. A new control algorithm based on supertwisting sliding mode for a gearless wind turbine by a permanent magnet synchronous generator (PMSG) is shown in [16]. The main features of this method are being chattering-free and its robustness against external disturbances such as grid fault conditions. The simulation results show better performance and robustness under different conditions for designed STSM controller. To solve the problem of the fault ride-through (FRT) capability and fault current issues, a nonlinear sliding mode controller (SMC) for the BFCL to enhance the FRT performance of the DFIG-based WT is proposed in [17]. Compared with other methods, this controller has robust performance in unpredicted voltage sag level and nonlinear features. Simulation results reveal that the SMC-based BFCL provides better performance compared with the conventional and PI controller-based BFCL to enhance the FRT. In [18], an interceptor can align itself with an impact angle frame in finite time, but this method has a drawback of the potential singularity problem when the error is a very small. In [19], a fast fixed-time second order sliding mode control method is presented for three-axis stabilized double slider mass moment missile. This method establishes the attitude dynamics model of the mass moment with complete coupling dynamics characteristics. For intercepting a manoeuvring target, a novel smooth adaptive fixed-time convergent nonsingular terminal sliding mode guidance law is designed in [20]. The sliding mode guidance control law designed in this paper has the characteristics of having no singularity and chattering. Though the paper designs an appropriate control law, it studies the manoeuvring target with engagement geometries and impact angles only. In [21], a new guidance law with impact angle constraints, which ensure finite-time convergence to a desired impact angle, was developed. In [22], the same singularity problem can also be found in a Finite-Time Guidance Law (FTGL) that only focuses on interception without considering impact angle constraints. Of course, there are also other methods for high manoeuvre flight especially in agile turn. But it is easy to find that although many methods have been proposed, they all just consider how to realize agile turn rather than considering multiple constraints in the process of agile turn. Therefore, it is meaningful to study the problem of agile turn with multiple constraints. Particularly, the process shows the engine reignition to guarantee the terminal velocity.

On the other hand, combining with the missile dynamic model and multiple constraints, the problem of agile turn can be regarded as a nonlinear optimal control problem. In terms of nonlinear optimal control problem, corresponding algorithms were also proposed in previous literatures. 
Reference [23] discusses the conditions on finite-time stability in time-delay systems and gives the whole process. At the same time, it introduces time-delay systems in the application of nonlinear systems. In [24], for the hyperchaotic Chen system, a piecewise spectral homotropy analysis method (PSHAM) is used for solving it. At the same time, an adaptive control approach and a parameter estimation update law are introduced for the hyperchaotic Chen system with completely unknown parameters. In [25], a fixed-time integral sliding mode controller based on fixed-time stability theory is proposed, which ensures precise convergence of the state variables for the system. The controller designed in this paper realizes the nonsingular chattering-free control of chaotic oscillation in nonlinear system. In [26], a smooth fixed-time convergent sliding mode controller is proposed, which is used for a missile flight system considering aerodynamic uncertainties. The drawback of this approach is that it does not introduce any discontinuous items to avoid the problem of chattering consequently. In addition, it also just considers the influence of aerodynamic force. The problem of a full-order and reduced-order unknown input observers for a particular class of time-delay systems is researched in [27]. It establishes the asymptotic stability and existence conditions for the designed observers. At the same time, the paper uses the quadruple-tank process as a benchmark to prove the efficiency of the proposed algorithms. For the uncertain systems with time-varying uncertainties, nonlinearities, and external disturbances, a linear matrix inequality-based second order sliding set control is proposed in [28]. Using this approach, the stability analysis can simplify the nonlinear system into a linear matrix inequality problem. The corresponding simulation results are displayed to show the effectiveness of the proposed approach. Reference [29] presents a controller in sense of the fixed-time concept for rigid spacecraft and the attitude of the spacecraft converging to the equilibrium. In [30], a global nonsingular terminal sliding mode control strategy for nonlinear systems is developed to overcome the singularity problem of terminal sliding mode control systems. Using this approach can guarantee the finite-time reachability of the systems to get the terminal sliding mode surface. In addition, to solve the dynamic load carrying capacity problems for mechanical manipulators, the optimal control theory was used in $[31,32]$. The aim of all these researches is to calculate the maximum-allowed load that a mechanical manipulator with flexible links can carry while traversing an optimal path. Moreover, to find the optimal trajectory of the flexible mobile manipulators in point-to-point motion, the optimal control approach is used in [33]. In this paper, the study emphasizes modelling of the complete optimal control problem by keeping all nonlinear states and costate variables as well as control constraints. The motions of articulated systems along specified paths are researched in [34]. Using optimal control theory, the paper establishes the optimization to minimize a time-energy cost function and the optimization problem is solved using the Pontryagin maximum principle.

Inspired by the previous discussion, the paper mainly focuses on proposing a new process of agile turn and establishing the missile dynamic model with deceleration parachute and two-stage engine. Compared with the traditional process, the new process can have a smaller turning radius, a shorter turning time, and a high terminal velocity. This scenario is necessary in air-air combat, attacking the concealed target in urban or the reverse side of the mountain and others. Firstly, considering the existence of two-stage engine and deceleration parachute, the paper defines the new process of agile turn and gives a detailed description for each phase. Then, several constraints in the real scenario are given in this paper. Combining all these conditions and some assumptions, a new dynamic model including two-stage engine and deceleration parachute is established. Then, using optimal control theory and monotonicity principle, the paper designs the nonlinear optimal control laws for each actuator for each phase with multiple constraints. At the same time, to ensure the terminal velocity, the paper gives the method to determine the optimal timing point of the second engine reignition based on optimal control theory, monotonicity principle, and the flight procedure of the missile.

In a brief, the main contribution of the research is that it proposes a new process of agile turn. Using optimal control theory, the paper designs the nonlinear optimal control law with multiple constraints for turning radius, turning time, and terminal velocity, which is different from previous works that just consider one of these constraints. Besides, using optimal control theory to solve the optimal intermediate instant is always complex. So, combining with monotonicity principle, the paper proposes an approach to determine the optimal intermediate state to ignite the secondary engine. At last, the rest of this paper is structured as follows. In Section 2, we give a brief introduction of the process for different types of agile turn. In Section 3, several constraints in the whole process of agile turn are presented. In Section 4, the nonlinear dynamic model of the missile is proposed, and the corresponding research problems are also given in this section. In this section, using optimal control theory, the optimal control laws and the optimal timing point of engine reignition of the missile are investigated. Several numerical simulations are reported in Section 5, which is followed by some concluding remarks in Section 6 . After "Conclusions" section, the future recommendation is added in Section 7 to further improve the work.

\section{Definition of the New Process of Agile Turn}

As we know, the traditional process of agile turn is that when leaving the launcher, the missile quickly enters the initial guidance phase. In this phase, through reaction jets or aerodynamic force or others, the missile soon completes pointing which is adjusted near the LOS. But, in the whole process, it does not care about the terminal velocity, the turning time, and the turning radius. The traditional process of agile turn is shown in Figure 1. The red line shows the variation of axial force.

As shown in Figure 1, it is obvious that the traditional process of agile turn just includes one phase. Once the missile leaves launcher, it does not care about the 


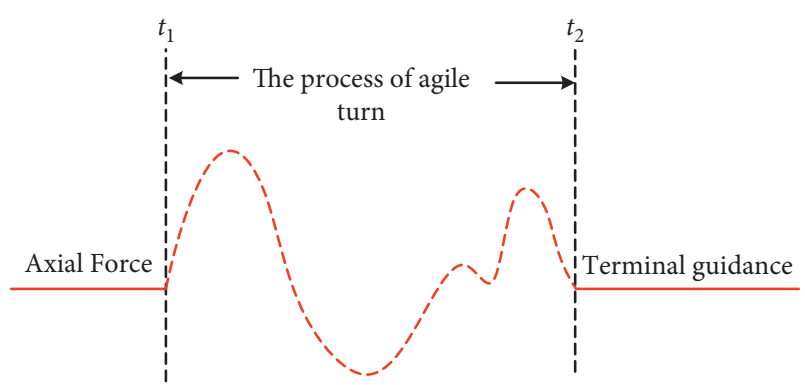

FIgURE 1: The traditional process of agile turn.

performance of agile turn. But, in the real combat scenario, it is widely known that the target does not only have the characteristics of great manoeuvring and high velocity. So, how to reduce the time of turning, decrease the turning radius, and have a high terminal velocity is very important. Therefore, this paper presents a new process of agile turn shown in Figure 2.

As shown in Figure 2, the new process of agile turn is divided into three phases called deceleration phase, turning phase, and engine reignition phase. When leaving the launcher, the missile enters the initial guidance phase. In this phase, the missile is affected by control force generated by air rudder, deceleration force, and aerodynamics force. When velocity decreased to a certain degree, the missile gets into larger angle manoeuvre phase. In this section, the missile accomplishes the large angle attitude manoeuvre affected by control force. At last, to ensure the terminal velocity, the secondary engine is ignited in the third phase.

\section{Constraints Description}

According to the real scenario, when carrying out the larger angle manoeuvre to attack the rear target, the concealed target in urban or the reverse side of the mountain and others, the process of agile turn includes so many constraints. Several major constraints will be introduced in this section.

3.1. Terminal Velocity Constraint. Figure 3 shows the missile attacking the rear target. To attack the high velocity target, the missile will be required to have a certain terminal velocity which is larger than the target's velocity. Then, the terminal velocity would be described as follows:

$$
v_{t f} \geq v_{t a r}
$$

where $v_{t f}$ is the terminal velocity after agile turn phase and $v_{\text {tar }}$ denotes the current target velocity.

3.2. Minimum Turning Radius Constraint. In the real combat scenario, which not only requires that the missile should have a high terminal velocity, but also accomplishes the large angle manoeuvre in a small space, the smaller the turning radius, the more the missile will have a broad application prospect, such as attacking the target which is closed to the mountain shown in Figure 3. So, considering this aspect, we require the turning radius to be smaller in large angle manoeuvre phase.

As shown in Figures 3 and 4, the turning radius should be smaller and the minimum turning radius can be described as follows:

$$
r \leq \min \left\{R_{\text {turn }}\{\ldots\}\right\}
$$

where $R_{\text {turn }}\{\ldots\}$ represents all the radii in each condition.

\section{Dynamic Model and Research Problems}

4.1. Nonlinear Dynamic Model. Before establishing the nonlinear dynamic model, several fundamental assumptions are introduced.

(1) The variation of static parameters like mass and moment of inertial through entire flight is not taken into consideration

(2) Trustworthy measurements of attitude angles and angular velocities are available with high precision sensors

(3) Aerodynamic uncertainties and their derivatives are bounded

(4) In deceleration phase, the parachute can be opened in a moment

(5) In engine reignition phase, the engine force just affects the direction of axis and without regard to the ignition time

(6) The thrust generated by reignition is defined as an impulse force

(7) The separation time between the missile and parachute is ignored

(8) The missile includes two-stage engine and the separation time between the primary engine and secondary engine is ignored

Based on all these assumptions, the dynamic model of the missile can be obtained, which is a strongly nonlinear and multivariable one. The model of the longitudinal dynamics of the missile shown in Figure 5 is given as follows, and the corresponding dynamic equation in longitudinal plane is shown in (3), which adds the deceleration force and the thrust generated by the secondary engine compared with the tradition equation [35].

As shown in Figure 5, the missile is made up of four parts mainly, including deceleration parachute, grey part of the primary engine, red part of the secondary engine, and the actuator. To achieve reignition, the missile will cast away the primary engine in a moment and ignite the secondary engine soon. In this paper, it does not consider the time of igniting. 


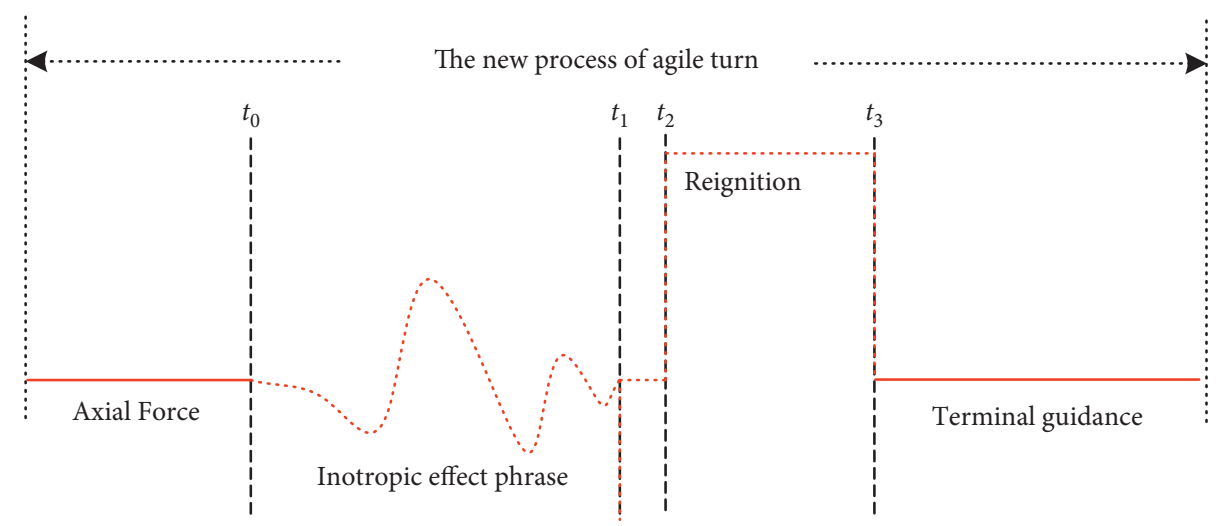

Figure 2: The new process of agile turn in this paper.

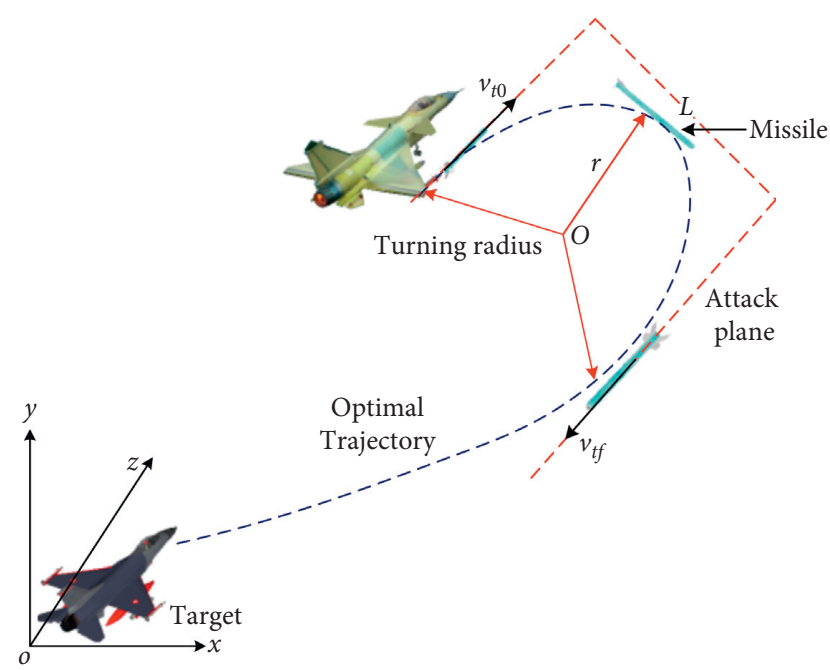

FIGURE 3: The terminal velocity constraint in agile turn.

$$
\left\{\begin{array}{l}
\dot{V}=\frac{P \cos \alpha-X-G \sin \theta-X_{x}+X_{\text {secthrust }}}{m} \\
\dot{\theta}=\frac{P \sin \alpha+Y-G \cos \theta}{m V} \\
\dot{w}_{z}=\frac{M_{z}^{\alpha} \alpha+M_{z}^{\bar{w}_{z}} \bar{w}_{z}+M_{z}^{\delta} \Delta \delta}{J_{z}} \\
\dot{\vartheta}=w_{z} \\
\dot{x}=V \cos \theta \\
\dot{y}=V \sin \theta \\
\alpha=\vartheta-\theta
\end{array},\right.
$$

where

$$
\left\{\begin{array}{l}
X=c_{x} q S \\
Y=c_{y} q S \\
M_{z}^{\alpha}=m_{z}^{\alpha} q S L \\
M_{z}^{\bar{w}_{z}}=m_{z}^{\bar{w}_{z}} q S L \\
M_{z}^{\delta}=m_{z}^{\delta} q S L \\
q=\frac{1}{2} \rho V^{2}
\end{array}\right.
$$

where $V$ denotes the velocity of the missile. $\theta$ denotes the trajectory inclination angle. $w_{z}$ is the pitch angular acceleration. $\vartheta$ is the pitch angle. $\alpha$ represents the attack angle. $m$ is the mass of the missile. $x$ and $y$ represent the position in longitudinal plane.

In addition, $Y$ is a function describing lift effects. $X_{x}$ denotes the dissertation force. $X_{\text {secthrust }}$ represents the secondary engine thrust, which is an impulsive force. The other control input of the model is $\Delta \delta$ which is the elevator's deflection. $X$ is the function denoting the aerodynamic force. $P$ represents the primary thrust force. $M_{z}^{\alpha}, M_{z}^{\bar{w}_{z}}$, and $M_{z}^{\delta}$ are the function providing the pitching moment. $G$ denotes the gravitational constant.

Other functions that appear in the previous state-space model are denoted as follows. $c_{x}$ is the lift coefficient. $c_{y}$ is the drag coefficient. $m_{z}^{\alpha}$ is the moment coefficient due to the attack angle. $m_{z}^{\bar{w}_{z}}$ is the moment coefficient due to damping and $m_{z}^{\delta}$ is the moment coefficient due to the elevator's deflection. $\rho$ is the density of air. $S$ is the reference area and $L$ is the mean aerodynamics cord.

Using state-space notation $x=\left[x_{1}, x_{2}, x_{3}, x_{4}, x_{5}, x_{6}\right]^{T}$, and defining the modified control input $u_{1}=X_{x}, u_{3}=\Delta \delta, u_{2}=X_{\text {secthrust }}$, one can obtain the following state-space description about the dynamics of the missile. 


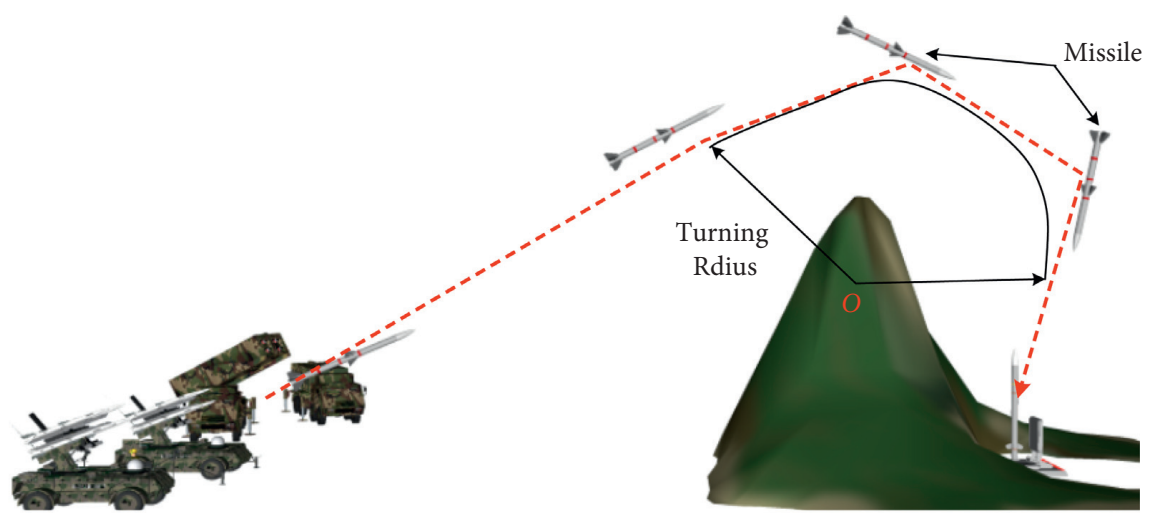

Figure 4: Attack the hidden target.

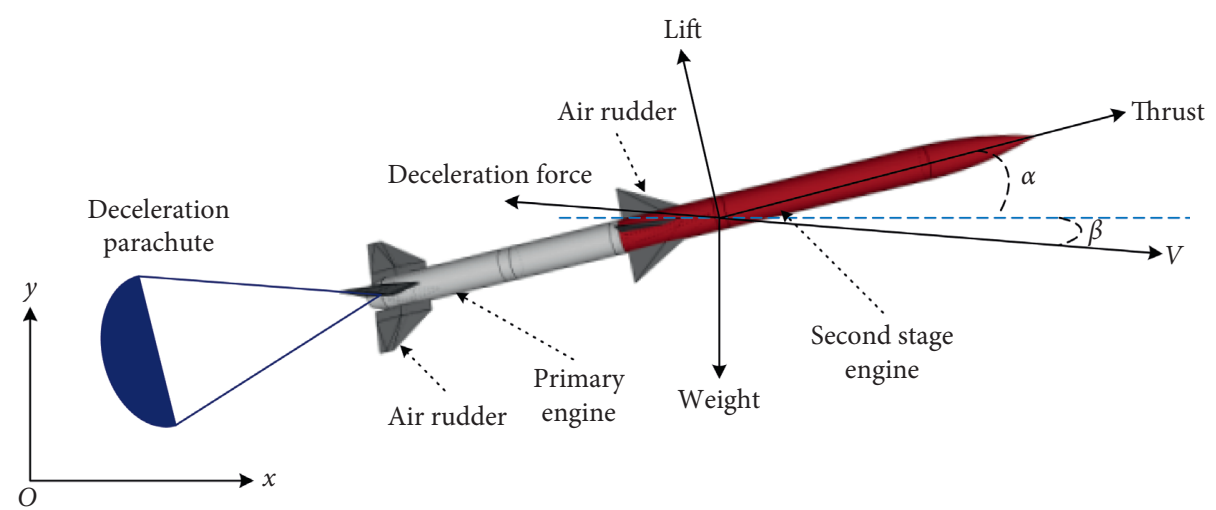

FIgURE 5: Diagram of the missile with deceleration parachute.

$$
\left\{\begin{array}{l}
\dot{x}_{1}=\frac{P \cos \left(x_{4}-x_{2}\right)}{m}-\frac{1}{2} \frac{1}{m} c_{x} \rho S x_{1}^{2}-\frac{G \sin x_{2}}{m}-\frac{u_{1}}{m}+\frac{u_{2}}{m} \\
\dot{x}_{2}=\frac{P \sin \left(x_{4}-x_{2}\right)}{m x_{1}}+\frac{1}{2} \frac{1}{m} c_{y} \rho S x_{1}-\frac{G \cos x_{2}}{m x_{1}} \\
\dot{x}_{3}=\frac{S L}{J_{z}} \frac{1}{2} \rho x_{1}{ }^{2}\left(m_{z}^{\alpha}\left(x_{4}-x_{2}\right)+m_{z}^{\bar{w}_{z}} \bar{w}_{z}+m_{z}^{\delta} u_{3}\right) \\
\dot{x}_{4}=x_{3} \\
\dot{x}_{5}=x_{1} \cos x_{2} \\
\dot{x}_{6}=x_{1} \sin x_{2}
\end{array}\right.
$$

where $x_{1}$ denotes the $V . x_{2}$ denotes the $\theta . x_{3}$ denotes the $w_{z}$. $x_{4}$ denotes the $9 . x_{5}$ denotes the $x$ in longitudinal plane. $x_{6}$ denotes the $y$ in longitudinal plane.

Then, the following functions are defined:

$$
\begin{gathered}
\left\{\begin{array}{l}
f_{1}=\frac{P \cos \left(x_{4}-x_{2}\right)}{m}-\frac{1}{2} \frac{1}{m} c_{x} \rho S x_{1}^{2}-\frac{G \sin x_{2}}{m} \\
g_{11}=-\frac{1}{m}, g_{12}=\frac{1}{m}, g_{13}=0
\end{array}\right. \\
\left\{\begin{array}{l}
f_{2}=\frac{P \sin \left(x_{4}-x_{2}\right)}{m x_{1}}+\frac{1}{2} \frac{1}{m} c x_{y} \rho S x_{1}-\frac{G \cos x_{2}}{m x_{1}}, \\
g_{21}=g_{22}=g_{23}=0
\end{array}\right. \\
\left\{\begin{array}{l}
f_{3}=\frac{S L}{J_{z}} \frac{1}{2} \rho x_{1}{ }^{2}\left(m_{z}^{\alpha}\left(x_{4}-x_{2}\right)+m_{z}^{\bar{w}_{z}} \bar{w}_{z}\right) \\
g_{31}=g_{32}=0, g_{33}=\frac{S L}{J_{z}} \frac{1}{2} \rho x_{1}{ }^{2} m_{z}^{\delta}
\end{array}\right.
\end{gathered}
$$




$$
\left\{\begin{array}{l}
g_{61}=g_{62}=g_{63}=0 \\
f_{6}=x_{1} \sin x_{2}
\end{array}\right.
$$

Consequently, the state-space description of the missile is written in the affine-in-the-input form.

$$
\left(\begin{array}{c}
\dot{x}_{1} \\
\dot{x}_{2} \\
\dot{x}_{3} \\
\dot{x}_{4} \\
\dot{x}_{5} \\
\dot{x}_{6}
\end{array}\right)=\left(\begin{array}{l}
f(x)_{1} \\
f(x)_{2} \\
f(x)_{3} \\
f(x)_{4} \\
f(x)_{5} \\
f(x)_{6}
\end{array}\right)+\left(\begin{array}{lll}
g(x)_{11} & g(x)_{12} & g(x)_{13} \\
g(x)_{21} & g(x)_{22} & g(x)_{23} \\
g(x)_{31} & g(x)_{32} & g(x)_{33} \\
g(x)_{41} & g(x)_{42} & g(x)_{43} \\
g(x)_{51} & g(x)_{52} & g(x)_{53} \\
g(x)_{61} & g(x)_{62} & g(x)_{63}
\end{array}\right)\left(\begin{array}{l}
u_{1} \\
u_{2} \\
u_{3}
\end{array}\right) \text {. }
$$

By denoting $x \in R^{6 \times 1}, u \in R^{6 \times 3}, f(x) \in R^{6 \times 1}$, and $g(x) \in R^{6 \times 3}$, then the previous state-space model can also be written in the concise form:

$$
\left\{\begin{array}{l}
\dot{x}=f(x)+G(x) u \\
\text { where }: G(x)=\left(\begin{array}{lll}
g(x)_{11} & g(x)_{12} & g(x)_{13} \\
g(x)_{21} & g(x)_{22} & g(x)_{23} \\
g(x)_{31} & g(x)_{32} & g(x)_{33} \\
g(x)_{41} & g(x)_{42} & g(x)_{43} \\
g(x)_{51} & g(x)_{52} & g(x)_{53} \\
g(x)_{61} & g(x)_{62} & g(x)_{63}
\end{array}\right),
\end{array}\right.
$$

where $\dot{x}$ represents the matrix of $\left[\dot{x}_{1}, \dot{x}_{2}, \dot{x}_{3}, \dot{x}_{4}, \dot{x}_{5}, \dot{x}_{6}\right]^{T}$. $f(x)$ represents the matrix of $\left[f(x)_{1}, f(x)_{2}, f(x)_{3}, f(x)_{4}, f(x)_{5}, f(x)_{6}\right]^{T}$.

4.2. Research Problems. In this section, we mainly discuss the optimal control problem with multiple constraints, such as terminal velocity and turning radius. At the same time, we will research how to determine the optimal time of the secondary engine ignition and give the corresponding process of proof. Firstly, the paper will design the optimal control law for different phases.

\subsubsection{Optimal Control Law Design}

(1) Deceleration Phase and Larger Angle Manoeuvre Phase. Firstly, during deceleration phase, the parachute is opened in a moment. It is mainly to reduce the velocity to a certain extent. Then, the missile will be soon accessed into the larger angle manoeuvre phase. As mentioned in previous section, the new process of agile turn mainly concerns the constraints of terminal velocity and turning radius in this paper. So, during these two phases, we will just consider the turning radius and take the minimum turning radius as a constraint. So, in this section, we will derive the optimal control laws of $u_{1}^{*}, u_{3}^{*}$ in deceleration phase and larger angle manoeuvre phase. The following chat of algorithm is shown in Figure 6.

Figure 6 shows the approximate flow of the algorithm in deceleration phase and larger angle manoeuvre phase to

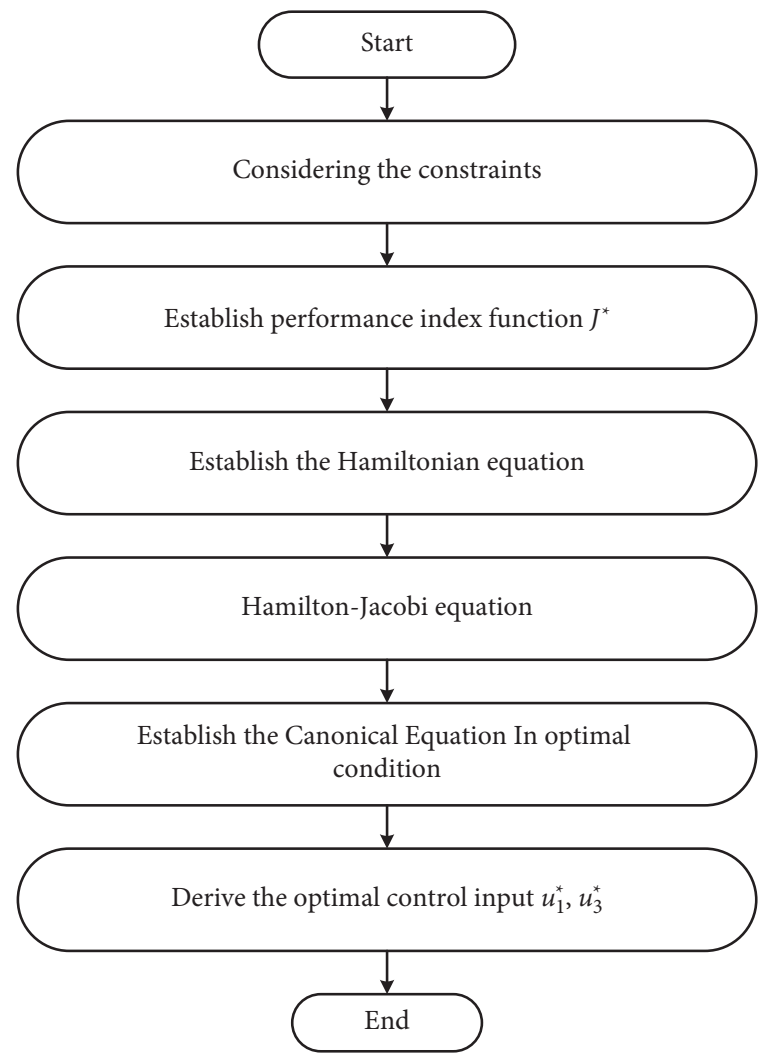

FIgURE 6: The approximate flow of algorithm.

derive the optimal control input $u_{1}^{*}, u_{3}^{*}$. Now, we will give the procedure of the algorithm in detail.

Theorem 1. The control laws equation (14) is the optimal control laws for different actuators during deceleration phase and larger angle manoeuvre phase of agile turn:

$$
\left\{\begin{array}{l}
u_{1}^{*}=g(x)_{11}\left(\frac{\tilde{r}}{\alpha g_{11}(x)+\tilde{f}_{1}(x)}\right)+\tilde{r},\left(t_{0}<t<t_{1}\right) \\
u_{3}^{*}=-g(x)_{33}\left(-f_{3}(x)+\gamma g_{33}(x)\right),\left(t_{0}<t<t_{1}\right)
\end{array} .\right.
$$

Remark 1. Before giving the process of derivation, the paper will declare that the secondary engine is not ignited during these two phases. Therefore, the missile is just affected by aerodynamic force, deceleration force, and control force generated by air rudder. So, we can ignore the thrust generated by the secondary engine. Then, we can obtain the following equation:

$$
u_{2}=X_{\text {secthrust }}=0 .
$$

Proof. As mentioned in previous section, during these two phases, the paper will just concern the turning radius. So, the corresponding constraint can be defined as follows: 


$$
r=\frac{1}{i} \sum_{t_{0}}^{t_{1}} r_{t_{i}}\left(t_{0}<t_{i}<t_{1}\right)
$$

where $t_{0}$ denotes the initial time. $t_{1}$ denotes the terminal moment, which is also the initial time during reignition phase. $r_{t_{i}}$ denotes the turning radius at $t_{i}$. From equation (16), it is obvious that, to make $r$ smallest, $r_{t_{i}}$ should be required to be smaller in each simulation step.

In general, the initial state of the missile is known, and it is defined as $x_{0}$. During these two phases, we just consider the turning radius rather than considering the state at $t_{1}$. So, we can regard this problem as two-point boundary value problem whose terminal time $t_{1}$ and the terminal state are free.

Therefore, according to the dynamic program theory and the turning radius constraint mentioned in previous section, the optimal cost function can be defined as follows:

$$
J^{*}=-\min _{u\left[t_{0}, t_{1}\right] \in \Omega}\left\{\int_{t_{0}}^{t_{1}} r \mathrm{~d} t+\frac{1}{2} \int_{t_{0}}^{t_{1}}\left(u_{1}^{2}+u_{3}^{2}\right) \mathrm{d} t\right\},
$$

where $r=x_{1} / \dot{x}_{2}$ denotes the turn radius. $u_{1}, u_{3}$ represent the deceleration force and the control force, respectively. by

For $J^{*}$, the corresponding Hamiltonian function is given

$$
H=r+\frac{1}{2}\left(u_{1}^{2}+u_{3}^{2}\right)+\lambda^{T}(f(x)+G(x) u),\left(t_{0}<t<t_{1}\right),
$$

where $\lambda^{T}(t)$ can be obtained as follows:

$$
\lambda^{T}(t)=\frac{\partial J^{*}}{\partial x} .
$$

According to equations (17) and (18), we can get the Hamilton-Jacobi equation as follows:

$$
-\frac{\partial J^{*}}{\partial x}=\min _{u} H=\min _{u}\left[r+\frac{1}{2}\left(u_{1}^{2}+u_{3}^{2}\right)+\lambda^{T}(f(x)+G(x) u)\right] .
$$

As mentioned in the previous section, in this paper the control inputs $u_{1}, u_{3}$ are unrestrained. At the same time, according to (15), we can obtain $u_{2}=0\left(t_{0}<t<t_{1}\right)$.

Then, according to equations (13) and (18), the optimal control input can be derived as follows at $t_{0}<t<t_{1}$ :

$$
\frac{\partial H}{\partial u}=\frac{\partial L}{\partial u}+\left(\frac{\partial f^{T}}{\partial u}\right) \frac{\partial J^{*}}{\partial x}=0,\left(t_{0}<t<t_{1}\right),
$$

where $(\partial L / \partial u),\left(\partial J^{*} / \partial x\right)$, and $\partial f^{T} / \partial u$ are as follows:

$$
\begin{aligned}
& \frac{\partial L}{\partial u}=\left[\begin{array}{c}
\frac{\partial L}{\partial u_{1}} \\
\frac{\partial L}{\partial u_{3}}
\end{array}\right]=\left[\begin{array}{c}
u_{1}+\frac{\partial r}{\partial u_{1}} \\
u_{3}+\frac{\partial r}{\partial u_{3}}
\end{array}\right],
\end{aligned}
$$

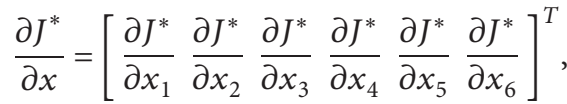

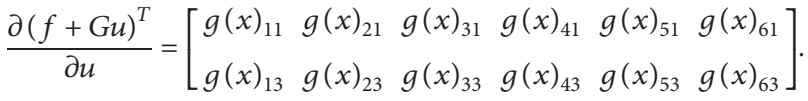

Combining with equations (21)-(24), the optimal controls $u_{1}, u_{3}$ can be obtained as follows:

$$
\left[\begin{array}{c}
u_{1}^{*} \\
u_{3}^{*}
\end{array}\right]=\left[\begin{array}{c}
g(x)_{11} \frac{\partial J^{*}}{\partial x_{1}}+\widetilde{r} \\
-g(x)_{33} \frac{\partial J^{*}}{\partial x_{3}}
\end{array}\right]
$$

According to equations (17) and (25), then we can obtain

$$
\begin{aligned}
\frac{\partial J^{*}}{\partial t}= & -\left\{L\left(x, u_{1}^{*}, u_{2}^{*}, t\right)\right. \\
& \left.+\left(\frac{\partial J^{*}}{\partial x}\right)^{T}\left(f(x)+G(x) u^{*}\right)\right\},\left(t_{0}<t<t_{1}\right) .
\end{aligned}
$$

As $\partial J^{*} / \partial t=0$, therefore, $\partial J^{*} / \partial x\left(t_{0}<t<t_{1}\right)$ can be derived:

$$
\begin{aligned}
r & +\frac{1}{2}\left(\left(u_{1}^{*}\right)^{2}+\left(u_{3}^{*}\right)^{2}\right) \\
& +\left(\frac{\partial J^{*}}{\partial x}\right)^{T}\left(f(x)+G(x) u^{*}\right)=0,\left(t_{0}<t<t_{1}\right) .
\end{aligned}
$$

According to (25), it is clear that $J^{*}$ is not including $x_{2}, x_{4}, x_{5}, x_{6}$. Then,

$$
\frac{\partial J^{*}}{\partial x_{2}}=\frac{\partial J^{*}}{\partial x_{4}}=\frac{\partial J^{*}}{\partial x_{5}}=\frac{\partial J^{*}}{\partial x_{6}}=0 \text {. }
$$

According to equations (26) and (27), we can obtain $\partial J^{*} / \partial x_{1}$ and $\partial J^{*} / \partial x_{3}$ :

$$
\left\{\begin{array}{l}
r+\frac{\left(u_{1}^{*}\right)^{2}}{2}+\frac{\partial J^{*}}{\partial x_{1}}\left(f_{1}(x)+g_{11}(x) u_{1}^{*}\right)=0 \\
\quad\left(t_{0}<t<t_{1}\right) . \\
\frac{\left(u_{3}^{*}\right)^{2}}{2}+\frac{\partial J^{*}}{\partial x_{3}}\left(f_{3}(x)+g_{33}(x) u_{3}^{*}\right)=0
\end{array}\right.
$$

Then,

$$
\left[\begin{array}{l}
\frac{\partial J^{*}}{\partial x_{1}} \\
\frac{\partial J^{*}}{\partial x_{3}}
\end{array}\right]=\left[\begin{array}{c}
\frac{\tilde{r}}{\alpha g_{11}(x)+\tilde{f}_{1}(x)} \\
-f_{3}(x)+\gamma g_{33}(x)
\end{array}\right]
$$

where $\alpha>0, \gamma$ denotes the scale factor and $\tilde{r}$ represents the partial derivatives of $u_{1}$.

Substituting equations (24) into (28), we can derive the optimal control inputs $u_{1}^{*}, u_{3}^{*}$ at $t_{0}<t<t_{1}$ : 


$$
\left[\begin{array}{l}
u_{1}^{*} \\
u_{3}^{*}
\end{array}\right]=\left[\begin{array}{c}
g(x)_{11}\left(\frac{\tilde{r}}{\alpha g_{11}(x)+\tilde{f}_{1}(x)}\right)+\tilde{r} \\
-g(x)_{33}\left(-f_{3}(x)+\gamma g_{33}(x)\right)
\end{array}\right],\left(t_{0}<t<t_{1}\right) .
$$

(2) Engine Reignition Phase. In this section, we will derive the optimal control laws $u_{1}^{*}, u_{2}^{*}$ in engine reignition phase. The following chat of algorithm is shown in Figure 7. As mentioned in previous section, to achieve engine reignition, the missile will cast away the parachute and the primary engine in a moment. To get the desired terminal velocity, the missile will soon ignite the secondary engine.

Figure 7 shows the approximate flow of the algorithm in engine reignition phase to derive the optimal control inputs $u_{1}^{*}, u_{2}^{*}$. Now, we will give the procedure of the algorithm in detail.

Theorem 2. Equation (32) is the optimal control law for different actuators during engine reignition phase of agile turn:

$$
\left\{\begin{array}{l}
u_{1}^{*}=g(x)_{11}\left(\frac{\tilde{r}}{\alpha g_{11}(x)+\widetilde{f}_{1}(x)}\right)+\widetilde{r},\left(t_{1}<t<t_{f}\right) \\
u_{2}^{*}=F,\left(t_{1}<t<t_{f}\right)
\end{array},\right.
$$

where $t_{f}$ denotes the terminal time. $t_{1}$ denotes the initial time, which is also the end time of larger angle manoeuvre phase. At the same time, $t_{1}$ is the time of the secondary engine ignition.

Remark 2. Before giving the process of derivation, the paper will declare that, to achieve engine reignition, the missile has casted away the primary engine and the deceleration parachute. Therefore, the missile is just affected by thrust generated by the secondary engine and control force generated by air rudder. So, we can ignore the thrust generated by the primary engine and the deceleration force. Then, we can get the following equation:

$$
u_{3}=0,\left(t_{1}<t<t_{f}\right) \text {. }
$$

Proof. During this phase, $x\left(t_{1}\right)$ denotes the initial state, and we can get the initial state $x\left(t_{1}\right)$ from larger angle manoeuvre phase. $x\left(t_{f}\right)$ denotes the terminal state. In this phase, we usually desire that the terminal velocity should be satisfied a certain value. So, this problem can also be regarded as a two-point boundary value problem, whose terminal time $t_{f}$ is free and the terminal state is constrained.

As mentioned in previous section, the terminal velocity must be satisfied at a certain value to attack the target. Therefore, the terminal state is usually constrained. The terminal velocity constraint can be defined as follows:

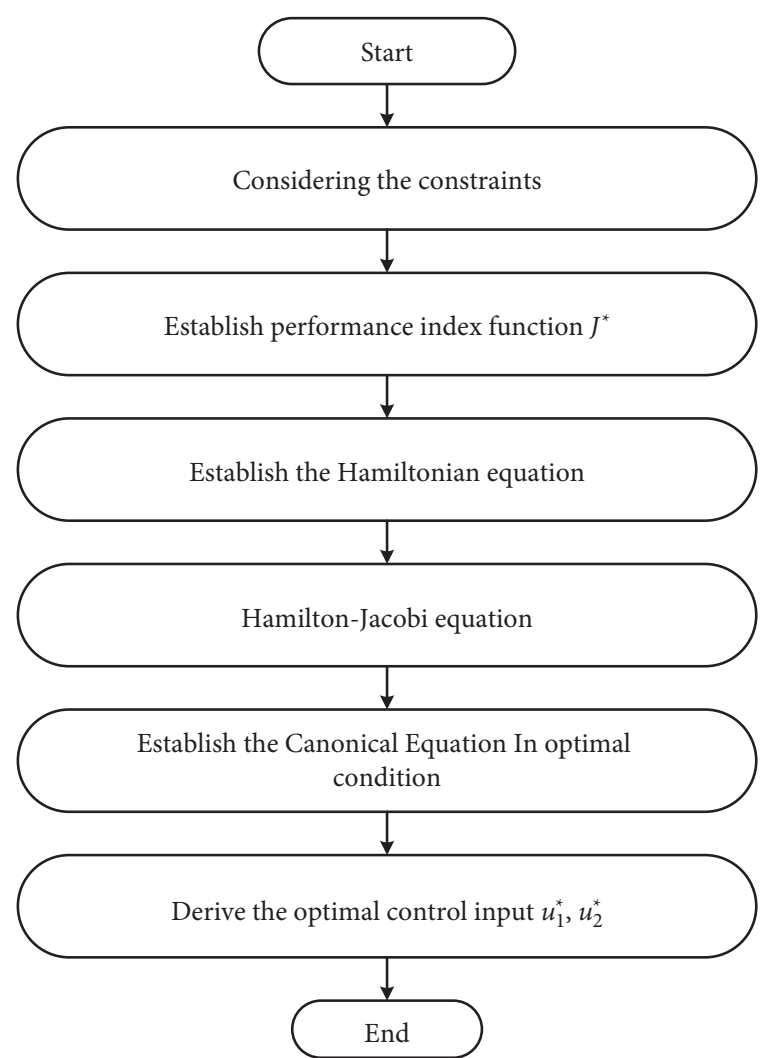

FIgURE 7: The approximate flow of algorithm.

$$
x_{1 t_{f}}-V_{\text {desir }}>0 \text {, }
$$

where $V_{\text {desir }}$ denotes the desired terminal velocity.

Therefore, considering terminal velocity and the turning radius constraint, the optimal cost function during this phase can be defined as follows:

$$
J^{*}=-\min _{u\left[t_{1}, t_{f}\right] \in \Omega}\left\{\Phi\left(x_{1 t_{f}}, t_{f}\right)+\int_{t_{1}}^{t_{\mathrm{f}}} r \mathrm{~d} t+\frac{1}{2} \int_{t_{1}}^{t_{\mathrm{f}}}\left(u_{1}^{2}+u_{2}^{2}\right) \mathrm{d} t\right\} .
$$

For $J^{*}$, the corresponding Hamiltonian is given by

$$
H=r+\frac{1}{2}\left(u_{1}^{2}+u_{2}^{2}\right)+\lambda^{T}(f(x)+G(x) u),\left(t_{1}<t<t_{f}\right),
$$

where $\lambda^{T}(t)$ can be obtained as follows:

$$
\lambda^{T}(t)=\frac{\partial J^{*}}{\partial x} .
$$

According to equations (36) and (37), we can get the Hamilton-Jacobi equation as follows:

$$
-\frac{\partial J^{*}}{\partial x}=\min _{u} H=\min _{u}\left[r+\frac{1}{2}\left(u_{1}^{2}+u_{2}^{2}\right)+\lambda^{T}(f(x)+G(x) u)\right] .
$$

In this paper, we consider that the control input $u_{1}$ is unrestrained and $u_{2}$ is an impulse force. There is no 
correlation between $u_{1}$ and $u_{2}$. Therefore, according to equations (13) and (36), $u_{1}$ can be derived as follows at $t_{1}<t<t_{f}$ :

$$
\frac{\partial H}{\partial u_{1}}=\frac{\partial L}{\partial u_{1}}+\left(\frac{\partial f^{T}}{\partial u_{1}}\right) \frac{\partial J^{*}}{\partial x}=0,\left(t_{1}<t<t_{f}\right)
$$

where $\left(\partial L / \partial u_{1}\right),\left(\partial J^{*} / \partial x\right)$, and $\partial f^{T} / \partial u_{1}$ are as follows:

$$
\begin{aligned}
\frac{\partial L}{\partial u_{1}} & =u_{1}+\frac{\partial r}{\partial u_{1}}, \\
\frac{\partial J^{*}}{\partial x} & =\left[\begin{array}{llllll}
\frac{\partial J^{*}}{\partial x_{1}} \frac{\partial J^{*}}{\partial x_{2}} & \frac{\partial J^{*}}{\partial x_{3}} & \frac{\partial J^{*}}{\partial x_{4}} & \frac{\partial J^{*}}{\partial x_{5}} & \frac{\partial J^{*}}{\partial x_{6}}
\end{array}\right]^{T}, \\
\frac{\partial(f+G u)^{T}}{\partial u_{1}} & =\left[\begin{array}{llllll}
g(x)_{11} & g(x)_{21} & g(x)_{31} & g(x)_{41} & g(x)_{51} & g(x)_{61} \\
g(x)_{13} & g(x)_{23} & g(x)_{33} & g(x)_{43} & g(x)_{53} & g(x)_{63}
\end{array}\right] .
\end{aligned}
$$

Combining with equations (39)-(42), the optimal control $u_{1}^{*}$ can be obtained as follows:

$$
u_{1}^{*}=g(x)_{11} \frac{\partial J^{*}}{\partial x_{1}}+\widetilde{r}
$$

According to equations (35), (38), and (43), then we can obtain

$$
\begin{aligned}
\frac{\partial J^{*}}{\partial t}= & \left\{L\left(x, u_{1}^{*}, u_{2}, t\right)\right. \\
& \left.+\left(\frac{\partial J^{*}}{\partial x}\right)^{T}\left(f(x)+G(x) u^{*}\right)\right\},\left(t_{1}<t<t_{f}\right) .
\end{aligned}
$$

As $\partial J^{*} / \partial t=0$, therefore, $\partial J^{*} / \partial x\left(t_{0}<t<t_{1}\right)$ can be derived:

$$
\begin{aligned}
r & +\frac{1}{2}\left(\left(u_{1}^{*}\right)^{2}+\left(u_{2}^{*}\right)^{2}\right) \\
& +\left(\frac{\partial J^{*}}{\partial x}\right)^{T}\left(f(x)+G(x) u^{*}\right)=0,\left(t_{0}<t<t_{1}\right) .
\end{aligned}
$$

According to equation (45), it is clear that $J^{*}$ is not including $x_{2}, x_{4}, x_{5}, x_{6}$. Then,

$$
\frac{\partial J^{*}}{\partial x_{2}}=\frac{\partial J^{*}}{\partial x_{4}}=\frac{\partial J^{*}}{\partial x_{5}}=\frac{\partial J^{*}}{\partial x_{6}}=0 .
$$

According to equations (45) and (46), we can obtain $\partial J^{*} / \partial x_{1}$ as follows:

$$
r+\frac{\left(u_{1}^{*}\right)^{2}}{2}+\frac{\partial J^{*}}{\partial x_{1}}\left(f_{1}(x)+g_{11}(x) u_{1}^{*}\right)=0\left(t_{1}<t<t_{f}\right) .
$$

Then,

$$
\frac{\partial J^{*}}{\partial x_{1}}=\frac{\tilde{r}}{\chi g_{11}(x)+\widetilde{f}_{1}(x)},
$$

where $\chi>0, \gamma$ denotes the scale factor and $\tilde{r}$ represents the partial derivatives of $u_{1}$.

Substituting equations (48) into (47), we can derive the optimal control input $u_{1}^{*}$ at $t_{1}<t<t_{f}$ :

$$
u_{1}^{*}=g(x)_{11}\left(\frac{\tilde{r}}{\chi g_{11}(x)+\widetilde{f}_{1}(x)}\right)+\widetilde{r},\left(t_{1}<t<t_{f}\right) .
$$

As mentioned in previous section, $u_{2}$ is an impulse force. Therefore, once the secondary engine is ignited, the engine should work and the thrust is equal to the max thrust of the engine. So, $u_{2}^{*}$ can be obtained as follows:

$$
u_{2}^{*}=F_{\mathrm{sec}},\left(t_{1}<t<t_{f}\right) \text {. }
$$

4.2.2. Determining the Time of Secondary Engine Ignition. In this section, we will determine the optimal timing point of secondary engine ignition $t_{1}$ in engine reignition phase. The following chat of algorithm is shown in Figure 8.

Figure 8 shows the approximate flow of the algorithm in determining the optimal timing point of secondary engine ignition $t_{1}$. Now, we will give the procedure of the algorithm in detail.

Theorem 3. The intermediate instant $t_{1}$ is the optimal time of the secondary engine ignition when the optimal control law $u_{1}^{*}=0$.

Proof. To prove the optimal time of the secondary engine ignition, the paper should demonstrate that the optimal control law $u_{1}^{*}$ is monotonically decreasing and it has the zero point. According to equation (27), the first derivative of $u_{1}^{*}$ is as follows:

$$
\zeta=g(x)_{11}\left(\frac{\dot{\vec{r}}\left(\alpha g_{11}(x)+\tilde{f}_{1}(x)\right)-\tilde{r}\left(\alpha \dot{g}_{11}(x)+\dot{\tilde{f_{1}}}(x)\right)}{\left(\alpha g_{11}(x)+\tilde{f}_{1}(x)\right)^{2}}\right)+\dot{\tilde{r}}
$$




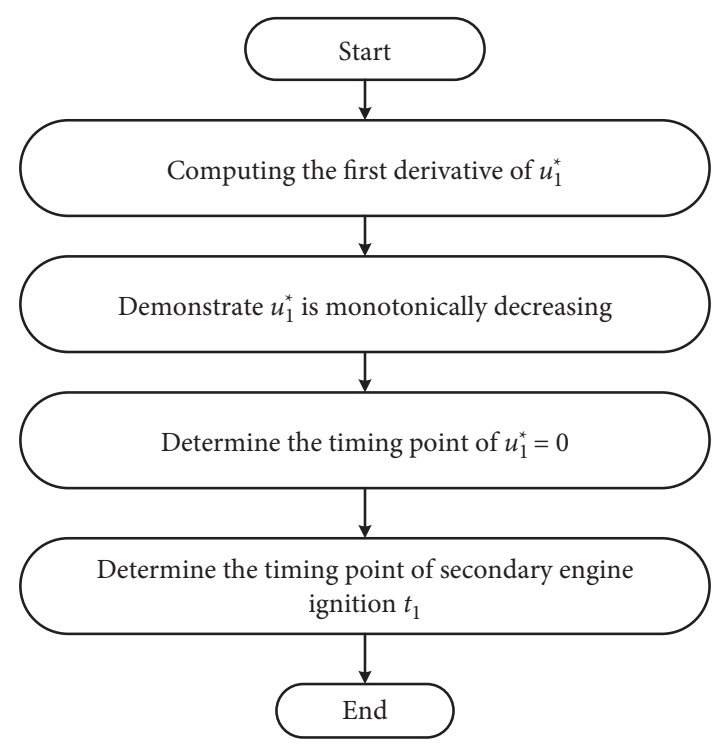

Figure 8: The approximate flow of determining $t_{1}$.

As mentioned in previous section, the turning radius $r=x_{1} /$ $\dot{x}_{2}$ and $\tilde{r}$ represents the partial derivatives of $u_{1}$. So, we can obtain the following equation.

$$
\left\{\begin{array}{l}
\tilde{r}=-\frac{g_{11}(x)}{\left(\dot{x}_{2}\right)^{2}}>0 \\
\dot{\tilde{r}}=\frac{2 g_{11}(x) \dot{f}_{2}}{\left(f_{2}\right)^{3}}<0
\end{array}\right.
$$

According to above section, where $\widetilde{f}_{1}(x)$ denotes the partial derivatives of $u_{1}$, therefore,

$$
\tilde{f}_{1}(x)=\left(-\frac{1}{2} \frac{1}{m} c_{x} \rho S x_{1}^{2}-\frac{1}{m}\right)<0 .
$$

According to equation (6) and $\alpha>0$, therefore,

$$
\frac{-\alpha}{m}+\left(-\frac{1}{2} \frac{1}{m} c_{x} \rho S x_{1}^{2}-\frac{1}{m}\right)<0 \text {. }
$$

Using equation (53), its first derivative can be written as follows:

$$
\dot{\overrightarrow{f_{1}}}(x)=-\frac{1}{2} \frac{1}{m} c_{x} \rho S x_{1} \dot{x}_{1}<0 .
$$

Therefore, combining equations (52)-(55), we can obtain the following equation to ensure $\zeta<0$.

$$
\left\{\begin{array}{l}
\left(\alpha g_{11}(x)+\widetilde{f}_{1}(x)\right)<0 \\
\tilde{r} \dot{\overrightarrow{f_{1}}}(x)<0
\end{array}\right.
$$

Therefore, according to equations (30)-(33), the optimal control $u_{1}^{*}$ has the corresponding zero point.

\section{Numerical Simulation}

To demonstrate the effectiveness of the control law designed in previous section, several numerical examples will be introduced in this section. Firstly, we use the shoulder-fired missile as an example. The parameters of the missile are listed in Table 1.

Case 1. In this simulation, it will just consider the turning radius. The initial parameters are presented as follows: the initial velocity is $V_{0}=200 \mathrm{~m} / \mathrm{s}$. The initial trajectory inclination angle is $\theta_{0}=50^{\circ}$. The initial position in longitudinal plane is presented $x_{0}=0 \mathrm{~m}, y_{0}=5 \mathrm{~m}$. And the target position is $x_{\mathrm{tar}}=500 \mathrm{~m}, y_{\mathrm{tar}}=5 \mathrm{~m}$. The integral step size is 0.001 s. In addition, using PSO (Particle Swarm Optimization) method as a contrast to illustrate the new process of angle turn is better. The simulation results are shown in Figures 9-12.

Figure 9 shows the ballistic curve by using optimal control method and PSO (Particle Swarm Optimization) method. As shown in Figure 9, it is obvious that, compared with PSO (Particle Swarm Optimization), the ballistic curve is smoother using optimal control method. Besides these, it is more important that the turning space is more less. The smaller the turning space that the missile requires, the wider the range of different applications used. This can also be demonstrated that the missile has a greater manoeuvrability.

Figures 10 and 11 give the variation of turning radius. It can be seen clearly that the turning radius of the missile is smaller than PSO (Particle Swarm Optimization) throughout all the procedure. It also can be seen that despite the turning radius showing the fluctuating phenomenon, the peak value is also smaller than PSO (Particle Swarm Optimization). The reason why this phenomenon appears is that it records the whole process of turning stage.

Figure 12 gives the variation of trajectory inclination angle. Compared with PSO (Particle Swarm Optimization) method, the trajectory inclination angle curve is smoother throughout the whole process. It is also obvious that the trajectory inclination angle falls more slowly in deceleration phase. After this, the change of trajectory inclination angle is relatively stable during terminal phase.

Figure 13 shows the changes of the missile velocity. From Figure 13, the velocity varies very slowly in whether the decreasing process or the ascending process. In addition, it is obvious that throughout the process the value of the minimum velocity is about three times bigger than PSO (Particle Swarm Optimization). At last, comparing with these two methods, we can also see that the terminal velocity is similar to PSO (Particle Swarm Optimization) method.

Table 2 gives the time consumption of the simulation in the same computer. It is obvious that when using optimal control method the time consumption is much less than PSO (Particle Swarm Optimization) method.

Table 3 gives the comparison of different aspects to make more explicit explanation and description by using different methods. It is obvious that throughout the process the turning radius is smaller and the minimum velocity is bigger, so that the turning time is shorter.

Case 2. In this simulation, it will consider the minimum turning radius, the optimal timing point of engine reignition, the terminal velocity, and the optimal control input. 
TABle 1: Parameters of the missile.

\begin{tabular}{lc}
\hline Parameter & Value \\
\hline Mass $(\mathrm{kg})$ & 15 \\
Reference area $\left(\mathrm{m}^{2}\right)$ & 0.0114 \\
Reference length $(\mathrm{m})$ & 1.027 \\
Diameter $(\mathrm{m})$ & 0.105 \\
Primary engine $(\mathrm{N})$ & 50 \\
Moment of inertia $\left(\mathrm{kg} \cdot \mathrm{m}^{2}\right)$ & 1.15 \\
\hline
\end{tabular}

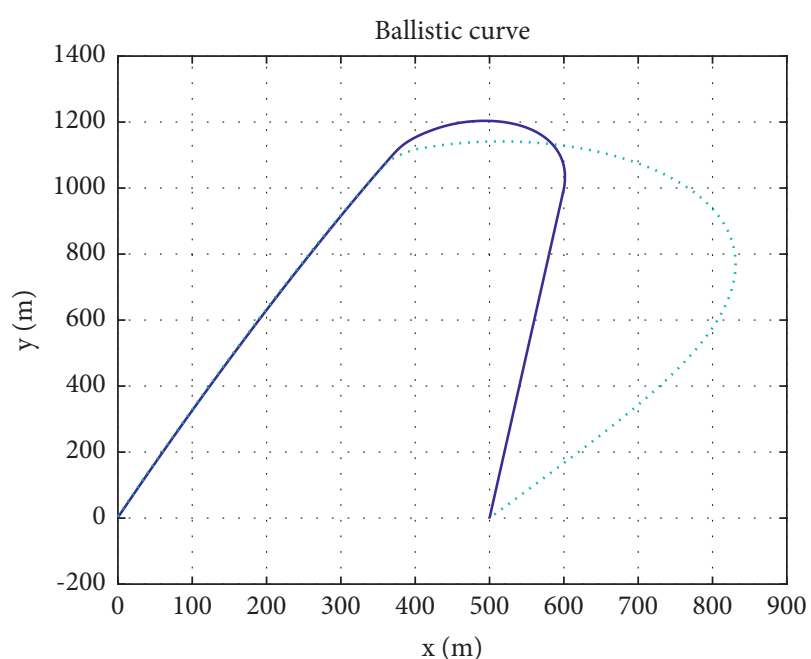

- Optimal Control Approach PSO

Figure 9: Ballistic curve of the missile.

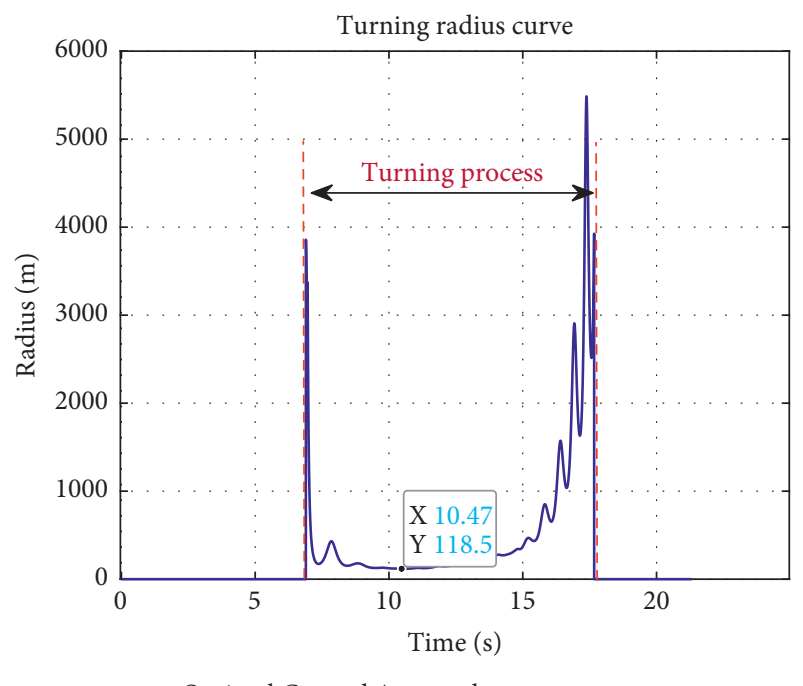

- Optimal Control Approach

FIGURE 10: The variation of the turning radius curve using optimal control.

The initial parameters are also presented as follows: the initial velocity is $V_{0}=200 \mathrm{~m} / \mathrm{s}$. The initial trajectory inclination angle is $\theta_{0}=70^{\circ}$. The initial position in longitudinal plane is presented as $x_{0}=0 \mathrm{~m}, y_{0}=5 \mathrm{~m}$. The target position

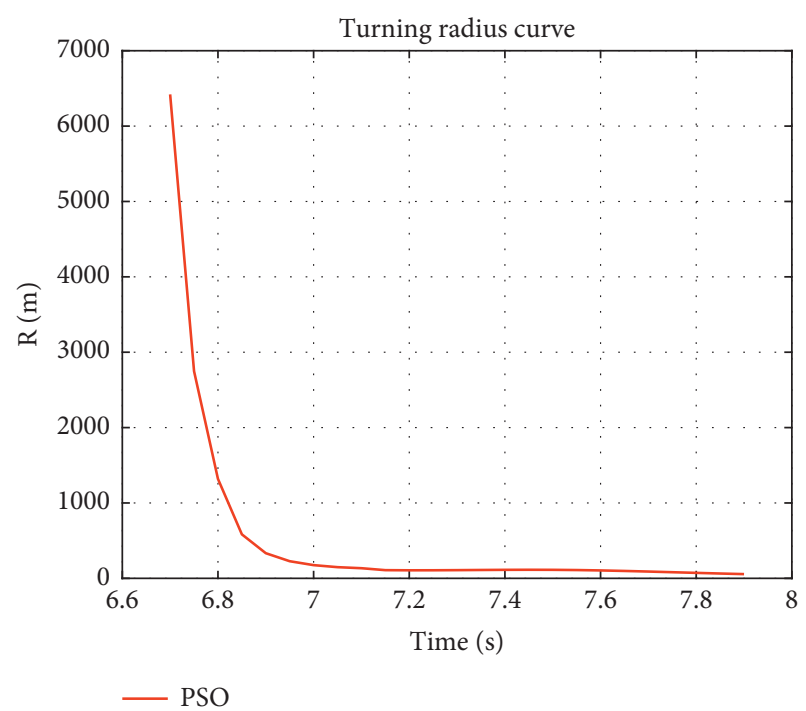

Figure 11: The variation of the turning radius curve using PSO (Particle Swarm Optimization).

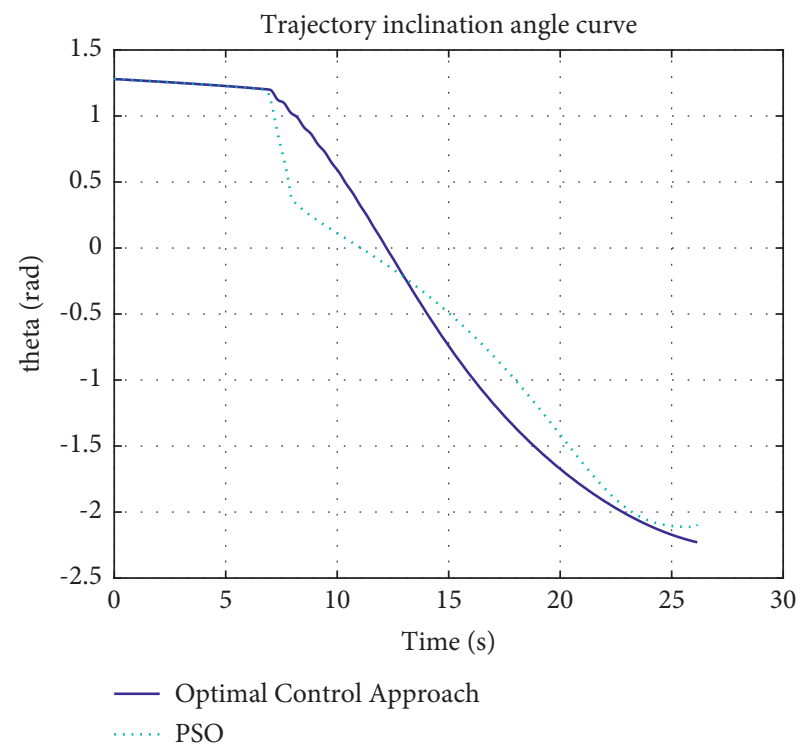

Figure 12: Trajectory inclination angle curve.

is $x_{\mathrm{tar}}=500 \mathrm{~m}, y_{\mathrm{tar}}=5 \mathrm{~m}$. The integral step size is also $0.001 \mathrm{~s}$. The simulation results are shown in Figures 14-21.

Figure 14 gives the variation of ballistic curve. With the same initial conditions, it is obvious that the maximum distance in $x$ and $y$ direction is larger compared with Figure 10. That is because the reignition thrust force is added in the process when the deceleration force decreased to zero. For this reason, the turning space also becomes larger.

Figure 15 shows the change of inclination angle. It is obvious that the variations of inclination angle are more sluggish especially between $0 \mathrm{~s}$ and $10 \mathrm{~s}$.

Figure 16 gives the velocity variations of the missile. It is pretty obvious that with the effectiveness of the engine reignition the minimum velocity in Figure 16 is greater than that in Figure 13. At the same time, it is obvious that the 


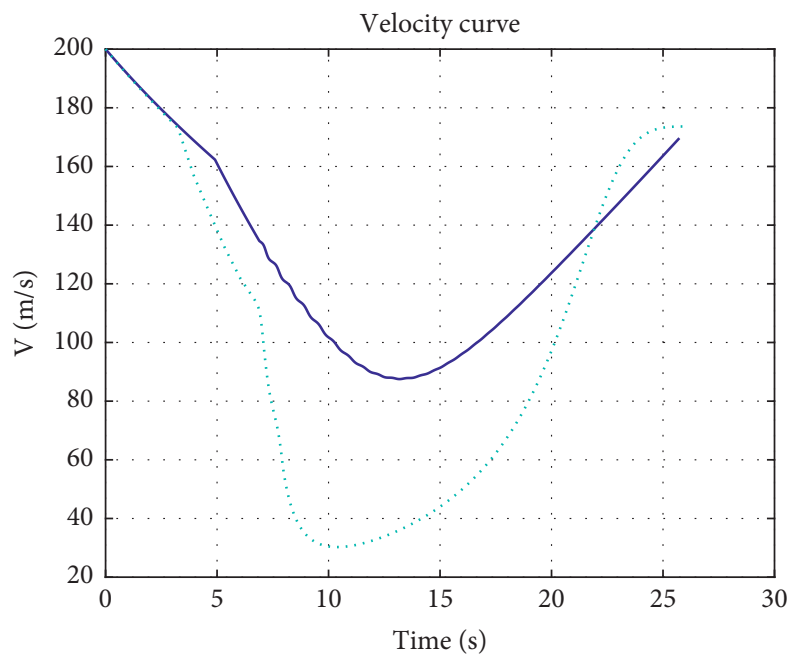

— Optimal Control Approach

PSO

Figure 13: The velocity curve of missile.

TABLE 2: The consumption of simulation time.

Method

Optimal control theory

Time

$8.056302 \mathrm{~s}$

Table 3: The comparison of different aspects.

\begin{tabular}{lcc}
\hline Aspects & Optimal control theory & PSO (Particle Swarm Optimization) \\
\hline Turning radius & $118.52 \mathrm{~m}$ & $640.31 \mathrm{~m}$ \\
Minimum velocity & $87.71 \mathrm{~m} / \mathrm{s}$ & $28.56 \mathrm{~m} / \mathrm{s}$ \\
Terminal velocity & $174.67 \mathrm{~m} / \mathrm{s}$ & $176.25 \mathrm{~m} / \mathrm{s}$ \\
\hline
\end{tabular}

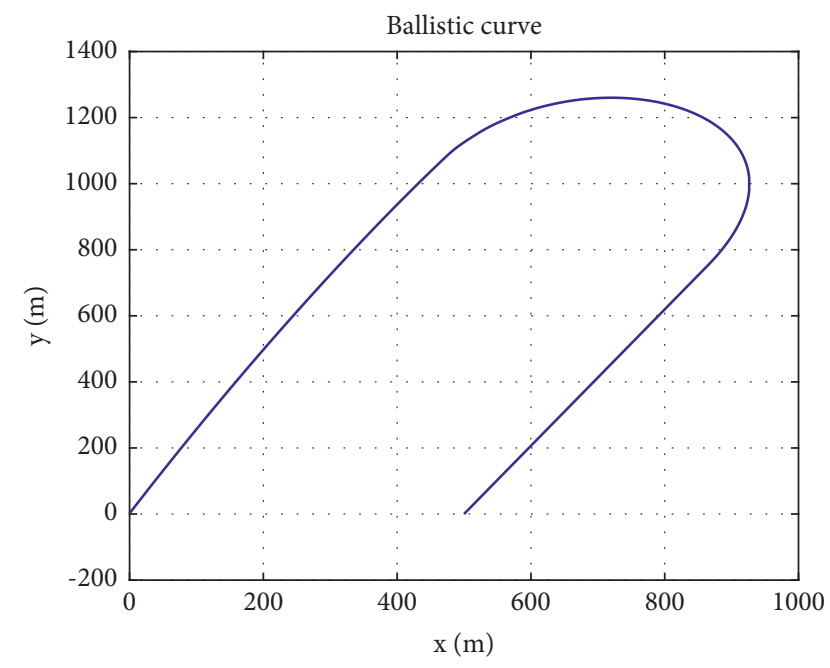

— Optimal Control Approach

Figure 14: Ballistic curve.

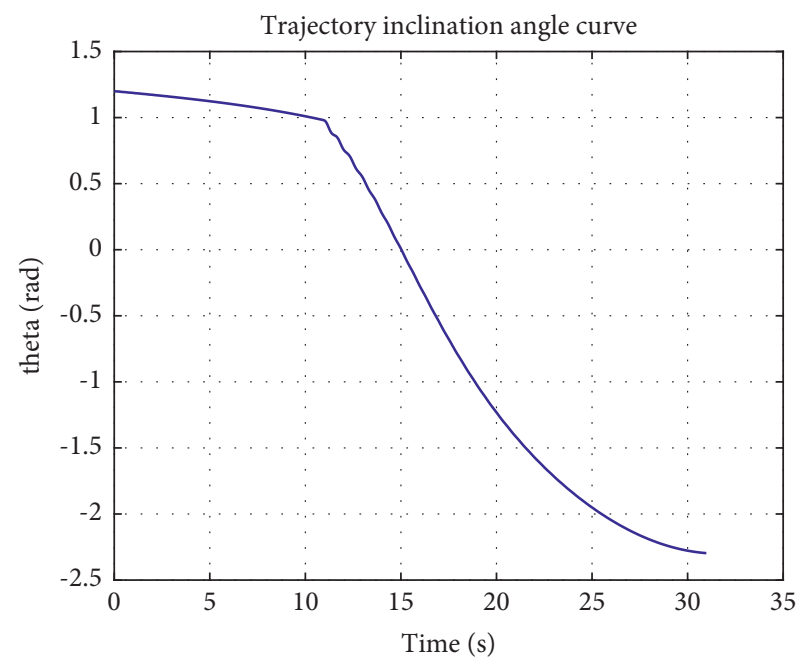

Optimal Control Approach

FIGURE 15: Trajectory inclination angle curve. 


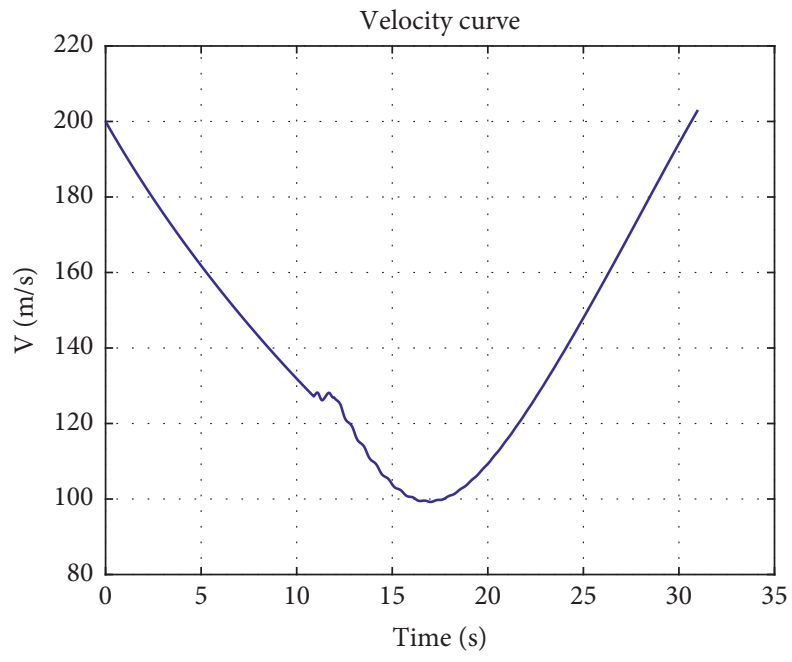

— Optimal Control Approach

FIGURE 16: The velocity curve of missile.

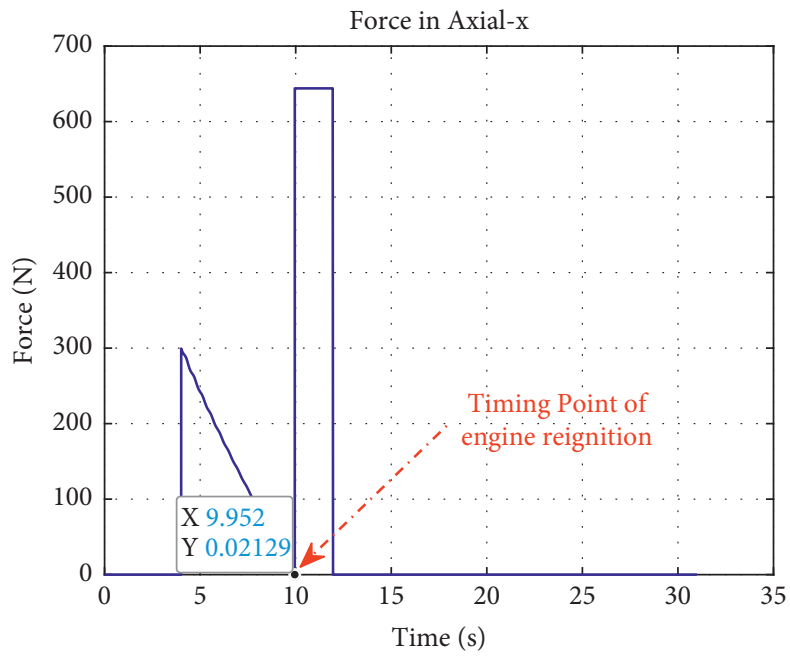

Figure 17: The force in axial- $x$ of missile.

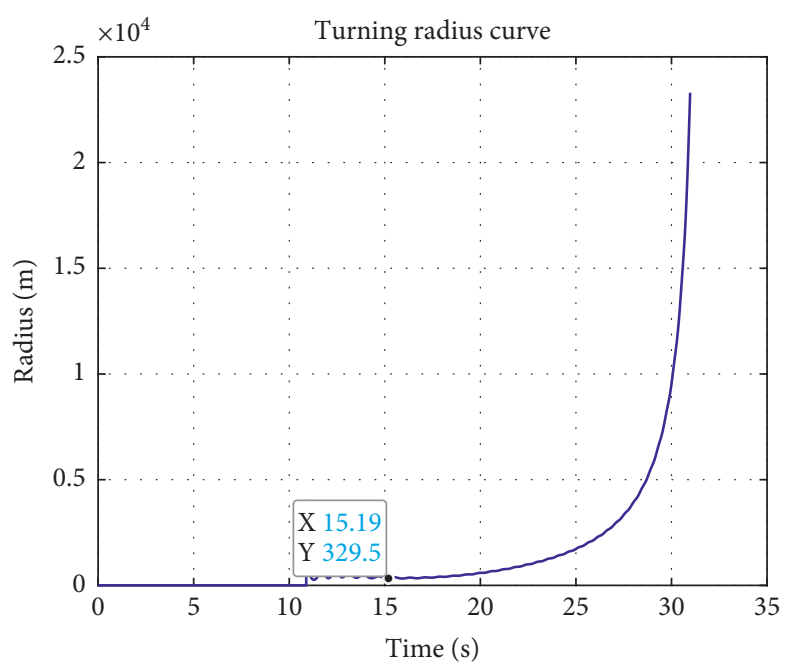

Optimal Control Approach

Figure 18: The variation of the turning radius curve. 


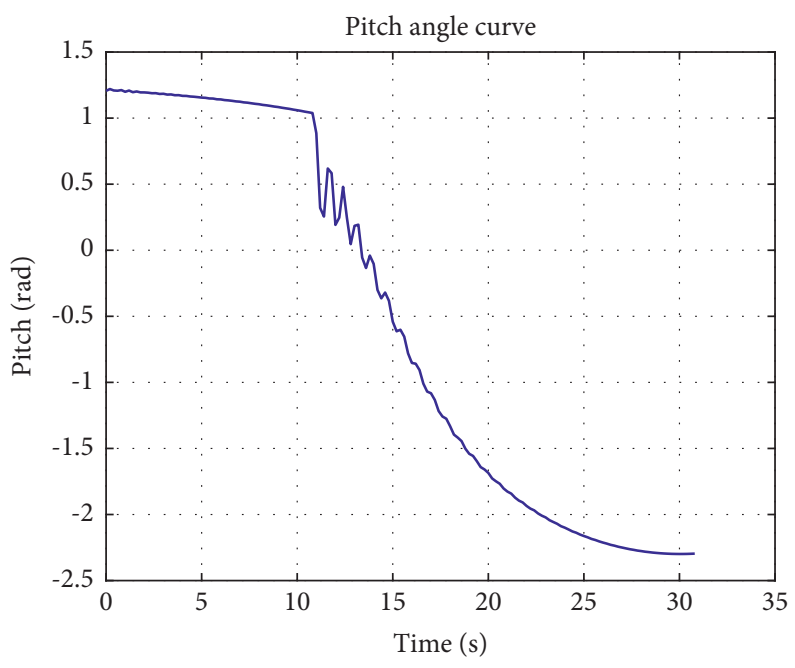

— Optimal Control Approach

Figure 19: The variation of pitch angle.

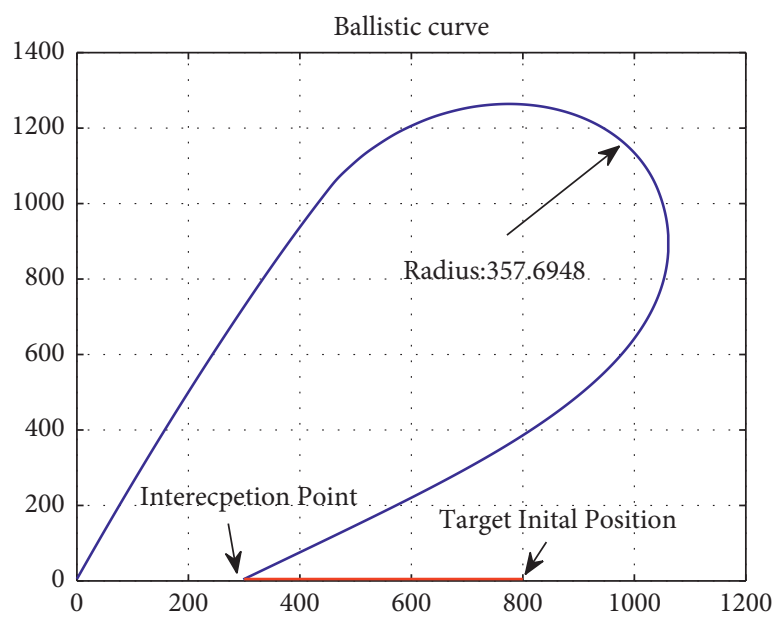

Figure 20: The pursuit attacking curve.

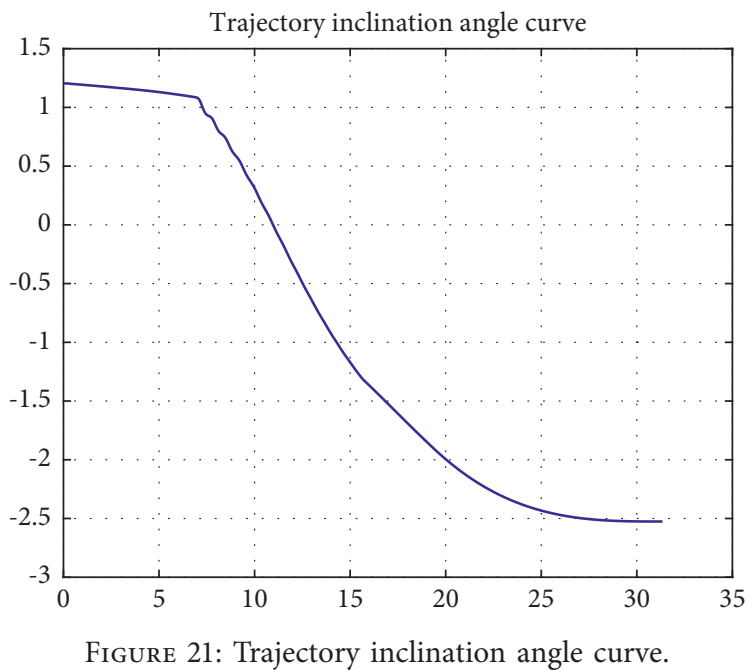


TABle 4: The comparison of different aspects.

\begin{tabular}{lccc}
\hline Aspects & Without engine ignition & With engine ignition & PSO \\
\hline Turning radius & $118.52 \mathrm{~m}$ & $324.9 \mathrm{~m}$ & $640.31 \mathrm{~m}$ \\
Minimum velocity & $87.71 \mathrm{~m} / \mathrm{s}$ & $99.87 \mathrm{~m} / \mathrm{s}$ & $28.56 \mathrm{~m} / \mathrm{s}$ \\
Terminal velocity & $174.67 \mathrm{~m} / \mathrm{s}$ & $196.25 \mathrm{~m} / \mathrm{s}$ & $176.25 \mathrm{~m} / \mathrm{s}$ \\
\hline
\end{tabular}

TABLE 5: The terminal manoeuvring target parameters.

\begin{tabular}{lc}
\hline Aspects & Value \\
\hline The start of manoeuvre time & $15.665 \mathrm{~s}$ \\
The end of manoeuvre time & $20.041 \mathrm{~s}$ \\
Terminal time & $5.624 \mathrm{~s}$ \\
Manoeuvre way & Horizontal manoeuvre \\
Manoeuvre velocity & $132 \mathrm{~m} / \mathrm{s}$ \\
\hline
\end{tabular}

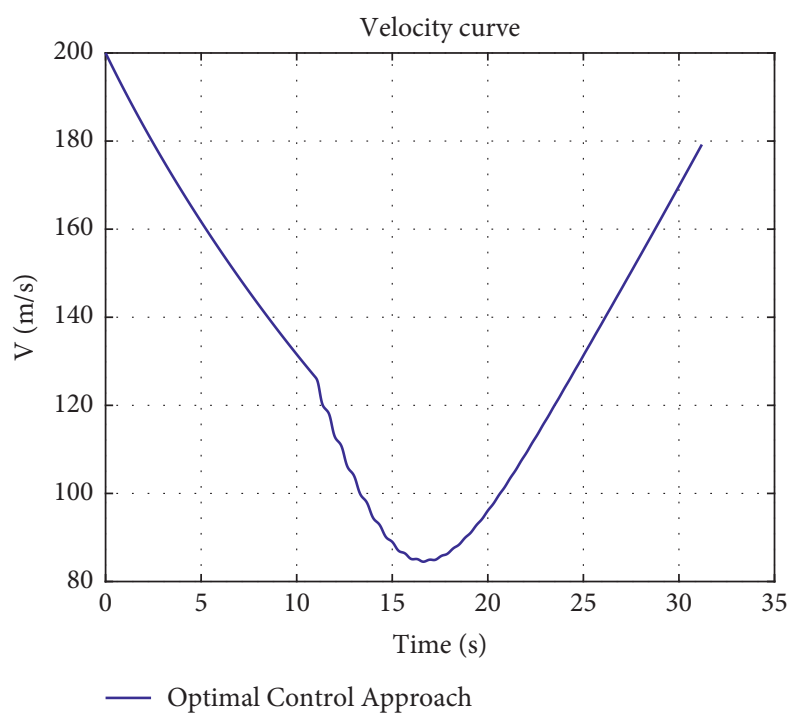

Figure 22: The velocity curve of missile.

missile also has a greater terminal velocity compared with Figure 13. Figure 17 shows the variation of the force in axial$x$ direction of the missile and the optimal timing point of engine reignition is at $9.552 \mathrm{~s}$.

Figure 18 describes the variation of the turning radius from deceleration phase to terminal guidance. Comparing with Figure 10, Figure 19 shows the variation of the pitch angle. Obviously, the jitter of the angle is very small, which demonstrates that the process of turning is relatively stable.

As shown in Table 4, it is obvious that with engine ignition the minimum velocity and the terminal velocity are all bigger than those without engine ignition and PSO (Particle Swarm Optimization) method. We will also find that the turning radius with engine ignition is bigger than that without engine ignition. The reason why the radius is increasing is that the engine reignition thrust is added in the process of agile turn, which increases the effectiveness of the axial thrust. But the turning radius value is also smaller than PSO method.
Case 3. Based on the constraint conditions of Case 2, we will consider the terminal manoeuvring target in this simulation. The initial parameters are also presented as follows: the initial velocity of the missile is $V_{0}=200 \mathrm{~m} / \mathrm{s}$. The initial trajectory inclination angle is $\theta_{0}=70^{\circ}$. The initial position in longitudinal plane is $x_{0}=0 \mathrm{~m}, y_{0}=5 \mathrm{~m}$. The target initial position is $x_{\mathrm{tar}}=800 \mathrm{~m}, y_{\mathrm{tar}}=5$ mand the manoeuvre parameters are shown in Table 5. The integral step size is also $0.001 \mathrm{~s}$. The simulation results are shown in Figures 20-23.

Figure 20 gives the pursuit attacking curve. It is obvious that missile can also attack the target, although the target has manoeuvrability. Compared with Figure 9, we will find that the maximum distance in $x$ and $y$ direction is larger. That is because the reignition thrust force and the initial position are all larger than Case 1. For this reason, the turning space also becomes larger.

Figure 21 shows the change of inclination angle. It is obvious that the variations of inclination angle are more 


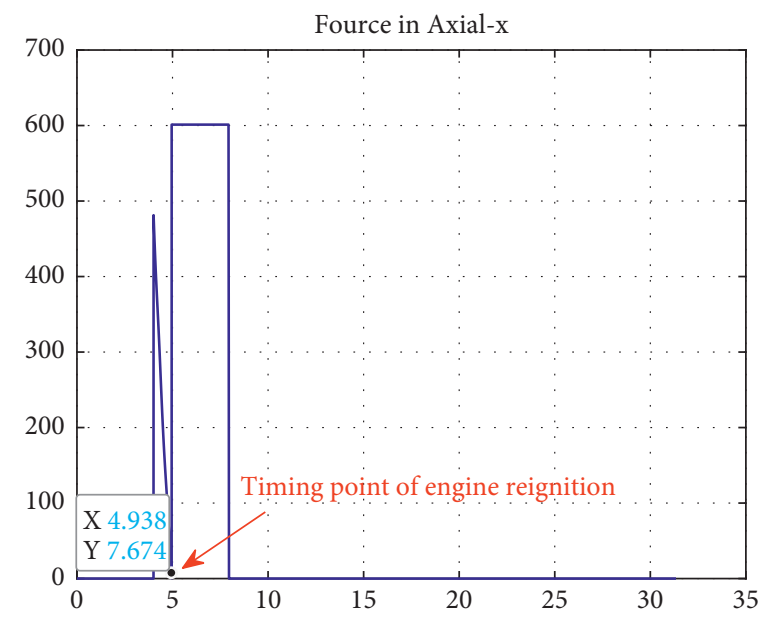

FIgURE 23: The force in axial- $x$ of missile.

TABLE 6: The comparison of different aspects.

\begin{tabular}{lcc}
\hline Aspects & With engine ignition & PSO \\
\hline Manoeuvres or not & YES & NO \\
Turning radius & $357.6948 \mathrm{~m}$ & $640.31 \mathrm{~m}$ \\
Minimum velocity & $86.72 \mathrm{~m} / \mathrm{s}$ & $28.56 \mathrm{~m} / \mathrm{s}$ \\
Terminal velocity & $180 \mathrm{~m} / \mathrm{s}$ & $176.25 \mathrm{~m} / \mathrm{s}$ \\
\hline
\end{tabular}

sluggish especially between $0 \mathrm{~s}$ and $10 \mathrm{~s}$ compared with the method of PSO (Particle Swarm Optimization).

Figure 22 gives the velocity variations of the missile. It is pretty obvious that with the effectiveness of the engine reignition the minimum velocity in Figure 22 is greater than PSO (Particle Swarm Optimization) method in Figure 13. At the same time, it is obvious that the missile also has a greater terminal velocity compared with Figure 13 . Figure 23 shows the variation of the force in axial- $x$ direction of the missile and the optimal timing point of engine reignition is at $4.938 \mathrm{~s}$.

As shown in Table 6, despite attacking the terminal manoeuvring target, the performance is also better than PSO (Particle Swarm Optimization) method. Moreover, it is obvious that with engine ignition the minimum velocity and the terminal velocity are all bigger than PSO method and the turning radius value is also smaller than PSO method.

\section{Conclusions}

A new process of agile turn with deceleration phase, larger angle manoeuvre phase, and the secondary engine ignition phase is proposed in this paper. Firstly, the paper describes the new process of agile turn. Then, it gives several main constraints' description. According to all these constraints and assumptions, the dynamic model including two-stage engine and deceleration parachute is established. After that, the paper gives the research problems. By using optimal control theory, the paper derives the optimal control laws for each actuator in different phase. In terms of determining the optimal timing point of the secondary engine ignition, the paper uses dynamic optimal control theory to derive it and gives the corresponding process of proof. At last, the paper takes PSO
(Particle Swarm Optimization) method as a contrast to demonstrate the effectiveness. According to several numerical simulations, it is obvious that the new process of agile turn is better than traditional process especially in having a smaller turning radius and a high terminal velocity.

\section{Future Recommendations}

Though the new process of agile turn is better than traditional process especially in having a smaller turning radius, a high terminal velocity decreases the time in turning phase. There exist many problems such as how to solve the problem of rigid-flexible coupling and how to eliminate the influence of the separation between the missile and parachute. We will continue researching all these problems in the future.

\section{Data Availability}

The data used to support the findings of this study are available from the corresponding author, if someone is interested.

\section{Conflicts of Interest}

The authors declare that they have no conflicts of interest.

\section{Acknowledgments}

The authors would like to thank the support of the flight control lab of Beijing University of Technology for this research. This study was supported by the National Natural Science Foundation of China (no. 61673212) and the Natural Science Foundation of Jiangsu Province (no. BK20161490).

\section{References}

[1] M. Mcfarland and A. Calise, "Neural-adaptive nonlinear autopilot design for an agile anti-air missile," in Proceedings of the Guidance Navigation, and Control Conference (AIAA), San Diego, CA, USA, July 1996.

[2] Y. Lu and S. Zhang, "An improvement on PWPF modulation of discrete RCS and design of the blended control logic," Acta 
Aeronautica et astronautica science, vol. 33, no. 9, pp. 1561-1570, 2012.

[3] P. Wang, W. Chen, H. Zou, and X. Yin, "Agile turn control considerations for air-to-air missile with reaction jets control system," Journal of Beijing University of Aeronautics and Astronautics, vol. 30, no. 5, pp. 395-399, 2005.

[4] L. Yan, C. Duan, G. Zhang, and Y. Zhao, "Research on the stability of an air-to-air missile lateral thrust and aerodynamic compound control system with a pulse modulator," Navigation Positioning and Timing, vol. 4, no. 1, pp. 42-48, 2017.

[5] G. Jie, S. Yongzhi, and L. Xiangdong, "Finite-time sliding mode attitude control for a reentry vehicle with blended aerodynamic surfaces and a reaction control system," Chinese Journal of Aeronautics, vol. 27, no. 4, pp. 964-976, 2014.

[6] S. Han, K. K. Min, M. J. Tahk, and Y. Y. Kim, "Velocity maximizing suboptimal control law for agile turn of air-to-air missile," in Proceedings of the16th International Conference on Control, Automation and Systems (ICCAS), Gyeongju, Korea, October 2016.

[7] A. Jajarmi, N. Pariz, S. Effati, and A. V. Kamyad, "Infinite horizon optimal control for nonlinear interconnected largescale dynamical systems with an application to optimal attitude control," Asian Journal of Control, vol. 14, no. 5, pp. 1239-1250, 2012.

[8] H. Roh, M. J. Tahk, J. Yun, and S. Park, "Guidance law for agile turn of air-to-air missile during boost phase," International Journal of Aeronautical and Space Sciences, vol. 18, no. 4, pp. 709-718, 2017.

[9] R. Furfaro and D. Mortari, "Least-squares solution of a class of optimal space guidance problems via theory of connections," Acta Astronautica, vol. 168, no. 3, pp. 92-103, 2020.

[10] A. Jajarmi and M. Hajipour, "An efficient recursive shooting method for the optimal control of time-varying systems with state time-delay," Applied Mathematical Modelling, vol. 40, no. 4, pp. 2756-2769, 2016.

[11] X. Wang, H. Zhang, S. Bai, and Y. Yue, "Design of agile satellite constellation based on hybrid-resampling particle swarm optimization method," Acta Astronautica, vol. 178, pp. 595-605, 2021.

[12] A. Jajarmi and D. Baleanu, "On the fractional optimal control problems with a general derivative operator," Asian Journal of Control, vol. 23, no. 2, pp. 1062-1071, 2021.

[13] G. Zhao, X. Han, Z. Hu, and C. Ma, "Integrated fuzzy sliding mode control-guidance for dual-control missiles," Control and Decision, vol. 31, no. 2, pp. 267-272, 2016.

[14] M. Omid, M. Saleh, and M.-H. Khooban, "Sliding mode disturbance observer control based on adaptive synchronization in a class of fractional-order chaotic systems," International Journal of Adaptive Control and Signal Processing, vol. 33, no. 3, pp. 462-474, 2019.

[15] J. Gao, "Studies on thickened tailings deposition in flume tests using the computational fluid dynamics (CFD) method," Canadian Geotechnical Journal, vol. 56, 2019.

[16] M. Nasiri, S. Mobayen, and Q. M. Zhu, "Super-twisting sliding mode control for gearless PMSG-based wind turbine," Complexity, vol. 2019, Article ID 6141607, 15 pages, 2019.

[17] M. Firouzi, M. Nasiri, M. Saleh, and G. B. Gharehpetian, "Sliding mode controller-based BFCL for fault ride-through performance enhancement of DFIG-based wind turbines," Complexity, vol. 2020, Article ID 1259539, 12 pages, 2020.

[18] Y. Zhang, S. Tang, and J. Guo, "An adaptive fast fixed-time guidance law with an impact angle constraint for intercepting maneuvering targets," Chinese Journal of Aeronautics, vol. 31, no. 6, 2018.
[19] J. L. Zhao, J. G. Guo, and J. Zhou, "Fixed-time second-order sliding mode control for mass moment missiles," Chinese Journal of Aeronautics, vol. 10, no. 40, pp. 1119-1223, 2019.

[20] F. Yang, K. Zhang, and L. Yu, "Adaptive super-twisting algorithm-based nonsingular terminal sliding mode guidance law," Journal of Control Science and Engineering, vol. 2020, no. 2, 11 pages, 2020.

[21] X. Zhang, M. Liu, and Y. Li, "Nonsingular terminal slidingmode-based guidance law design with impact angle constraints," Iranian Journal of Science and Technology, Transactions of Electrical Engineering, vol. 43, no. 1, pp. 47-54, 2019.

[22] L. Sun, W. Wang, R. Yi, and S. Xiong, "A novel guidance law using fast terminal sliding mode control with impact angle constraints," ISA Transactions, vol. 64, pp. 12-23, 2016.

[23] D. Efimov, A. Polyakov, E. Fridman, W. Perruquetti, and J.-P. Richard, "Comments on finite-time stability of timedelay systems," Automatica, vol. 50, no. 7, pp. 1944-1947, 2014.

[24] S. Effati, H. Saberi Nik, and A. Jajarmi, "Hyperchaos control of the hyperchaotic Chen system by optimal control design," Nonlinear Dynamics, vol. 73, no. 1-2, pp. 499-508, 2013.

[25] J. Wang, C. Liu, Y. Wang, and G. C. Zheng, "Fixed time integral sliding mode controller and its application to the suppression of chaotic oscillation in power system," Chinese Physics B, vol. 27, no. 7, 2018.

[26] Y. Yun, J. Guo, and S. Tang, "Robust smooth sliding-modebased controller with fixed-time convergence for missiles considering aerodynamic uncertainty," International Journal of Aerospace Engineering, vol. 2018, Article ID 9875462, 12 pages, 2018.

[27] S. Ben Warrad, O. Boubaker, M. Lungu, and S. Mobayen, "Full and reduced-order unknown input observer design for linear time-delay systems with multiple delays," Mathematical Problems in Engineering, vol. 2018, Article ID 1745734, 13 pages, 2018.

[28] J. Maryam and M. Saleh, "Second-order sliding set design for a class of uncertain nonlinear systems with disturbances: an LMI approach," Mathematics and Computers in Simulation, vol. 156, p. S0378475418301733, 2018.

[29] B. Jiang, Q. Hu, and M. I. Friswell, "Fixed-time attitude control for rigid spacecraft with actuator saturation and faults," IEEE Transactions on Control Systems Technology, vol. 24, no. 5, pp. 1892-1898, 2016.

[30] Y. Feng, X. Yu, and F. Han, "On nonsingular terminal slidingmode control of nonlinear systems," Automatica, vol. 49, no. 6, pp. 1715-1722, 2013.

[31] L. T. Wang and B. Ravani, "Dynamic load carrying capacity of mechanical manipulators-Part II: computational procedure and applications," Journal of Dynamic Systems, Measurement, and Control, vol. 110, no. 1, pp. 53-61, 1988.

[32] A. M. Shafei and M. H. Korayem, "Theoretical and experimental study of dynamic load-carrying capacity for flexible robotic arms in point-to-point motion," Optimal Control Applications and Methods, vol. 38, no. 6, pp. 963-972, 2017.

[33] M. H. Korayem, H. R. Nohooji, and A. Nikoobin, "Path planning of mobile elastic robotic arms by indirect approach of optimal control," International Journal of Advanced Robotic Systems, vol. 8, no. 1, pp. 10-20, 2011.

[34] Z. Shiller, "Time-energy optimal control of articulated systems with geometric path constraints," IEEE International Conference on Robotics and Automation, vol. 4, pp. 2680-2685, 1994.

[35] X. F. Qian, R. X. Lin, and Y. N. Zhao, "Missile flight aerodynamics Part 2," Beijing institute of technology press, vol. 1, no. 11, pp. 28-52, 202. 